\title{
Systematic Pan-Cancer Analysis and Experimental Verification Identify FOXA1 As An Immunological and Prognostic Biomarker In Epithelial Ovarian Cancer
}

\author{
Kai Wang \\ Wenzhou Medical University \\ Junhui Yu \\ Wenzhou Medical University \\ Chenan Guan \\ Wenzhou Medical University \\ Xing Chen \\ Wenzhou Medical University \\ Xianwen Shang \\ Wenzhou Medical University \\ Shuangshuang Mei \\ Wenzhou Medical University \\ Xingjun Feng \\ Wenzhou Medical University \\ Lingzhi Zheng ( $\nabla$ zhenglingzhi2000@126.com ) \\ Wenzhou Medical University https://orcid.org/0000-0002-8422-6052
}

Primary research

Keywords: Epithelial ovarian cancer, FOXA1, EMT, TCGA, pan-cancer

Posted Date: May 12th, 2021

DOI: https://doi.org/10.21203/rs.3.rs-488513/v1

License: (c) (i) This work is licensed under a Creative Commons Attribution 4.0 International License. Read Full License 


\section{Abstract}

Background: Epithelial ovarian cancer (EOC) has the lowest survival rate among female reproductive cancers present with symptoms of aggressive malignancies, poor prognosis, drug resistance and postoperative recurrence. The majority of patients with EOC are diagnosed at an advanced stage due to the therapeutic challenges including lack of early diagnosis and effective therapeutic targets for EOC.

Methods: Pan-cancer analyses were performed to explore the features of Forkhead box (FOX) A1 (FOXA1) using data from TCGA and GTEx database. R package "clusterprofiler" was used to perform the enrichment analysis of FOXA1 in EOC. Data downloaded from Drug Sensitivity in Cancer (GDSC) database were used to evaluate the association between FOXA1 and anti-tumor drug sensitivity. In experimental verification, FOXA1 expression was detected using qRT-PCR and Western blot assays. Western blot, immunofluorescence staining and transwell assays were used to assess the influence of FOXA1 silencing on epithelial-mesenchymal transition (EMT) of EOC cells

Results: We found that FOXA1 was highly expressed in EOC and predicted poorer survival of EOC patients. We observed that FOXA1 expression was positively correlated EMT related pathways. Through experimental verification, we found the underlying function of FOXA1 to promote EMT in ovarian cancers. Results from western blot, immunofluorescence staining and transwell assays showed that FOXA1 silencing impeded the progression of EMT and invasiveness of the cancer cells. Furthermore, CCK-8 and invasion assays suggested that siRNA-FOXA1 attenuated the ability of cancer cells to metastasize and proliferate. Dual-luciferase reporter assays confirmed the binding activity of FOXA1 to the promoter of connective tissue growth factor (CTGF). In addition, we found that FOXA1 was closely correlated immunosuppressive microenvironment of EOC. High FOXA1 expression may contribute to resistance of many anti-cancer drugs.

Conclusions: Our results predict and validate the function of FOXA1 in promoting EMT and the progression of disease in EOC. Targeting FOXA1 may improve the sensitivity of EOC treatment.

\section{Introduction}

Ovarian cancer is a fatal gynecological cancer [1-3]. Ovarian cancer is often identified in the advanced phase because it lacks early-stage symptoms. Even the symptoms in the advanced stage, including abdominal pain and swelling, loss of appetite, and frequent urination, can be overlooked. Moreover, ovarian cancer is genetically and phenotypically heterogeneous without reliable and effective biomarkers for diagnosis and prognosis, which also makes it challenging to detect ovarian cancer in the early phase [4]. The 5-year survival rate of patients with advanced ovarian cancer was increased with the development of surgery techniques and combined therapies of chemotherapy drugs and monoclonal antibody drugs. However, the long-term survival rate still remains disappointing [5, 6]. Further investigating the mechanisms of the occurrence and progression of ovarian cancer is fundamental for diagnosis in the early stage and therapy of ovarian cancers. 
Epithelial-to-Mesenchymal Transition (EMT) has long been considered related to tumorigenesis, in which the differentiated epithelial cells (E) shift towards mesenchymal-like (M) properties by losing the apicalbasal polarity and cell-cell adhesion, and gaining the properties of stemness, migration and proliferation. During the development of carcinoma, EMT leads to augmented capability to invade, migrate, resist to chemotherapy drugs and metastasize in neoplastic cells[7]. In the occurrence of EMT, E-cadherin is downregulated because E-cadherin-mediated intercellular tight junctions are destroyed [8]. Moreover, EMT is often characterized by a gained motility and changed morphology of the cells as well as the compositional alteration of cytoskeleton filaments [9]. An overexpressed intermediate filament protein, vimentin, is commonly observed in various epithelial cancers, including EOC. Thereby, mesenchymal markers are often upregulated in EMT, including matrix metallopeptidases (MMPs), fibronectin, vimentin and $\mathrm{N}$-cadherin, while epithelial markers are downregulated, such as claudins and E-cadherin[10].

EMT, a complicated biological process, plays a key role in an array of physiological and pathological events, including tumor progression [11, 12], cancer cell invasion, embryogenesis [13, 14], wound healing [15], fibrosis[16, 17], therapy resistance [18], inflammation [19], and etc. There are various signaling pathways have participated in mediating EMT, in which TGF- $\beta$ pathway is considered closely related to the invasion-metastasis cascade of EOC by enhancing the stemness of cancer stem cells, and promoting the invasiveness and migration of tumor cells [20].

Recently, the transcription factor forkhead-box A1 (FOXA1) was reported to be a meaningful EMT reporter [21]. The overexpression of FOXA1 was also reported in several cancer types, and, thus, it serves as a significant indicator of the poor overall survival (OS) of patients. FOXA1 belongs to FOX family, participating in the development of many endoderm-derived organs mainly through mediating the nuclear steroid receptor signaling $[22,23]$. It has been studied in various human cancers, including thyroid cancer [24], breast cancer [25], lung cancer [26], gastric cancer [27], and prostate cancer [28], and suggested to be closely associated with the malignancy and clinicopathological characteristics of tumors..

Yet, there is still insufficient work to illustrate the mechanism regarding the promotive effects of FOXA1 on EMT and further tumor microenvironment (TME) in EOC. In the study, we first explored the pan-cancer features of FOXA1. EMT and immune related pathways were closely correlated with FOXA1 in EOC. In the aspect of experimental verification, we compared the expression in different EOC cell lines. Cell proliferation and invasion were examined following siRNA-FOXA1 transfection to demonstrate the role of FOXA1 in altering cell towards EMT-like characteristics and in promoting tumorigenesis in EOC. More importantly, the interaction between FOXA1 and connective tissue growth factor (CTGF) is of our great interest, as the preliminary study reported that FOXA1 may affect the expression of CTGF [29]. Thereby, it is hypothesized that the transcription factor, FOXA1, may directly regulate the transcription of CTGF by interacting with its promoter region.

CTGF is implicated in multiple complexed signaling networks and involved in various biological processes, including cell adhesion, migration, proliferation, angiogenesis, and extracellular matrix synthesis [30]. Although the pleiotropic bio-functions of CTGF remain to be elucidated, researchers have 
noted its association with EMT and cancer malignancies. CTGF is preferentially expressed in aggressive neoplasms [31]. The induction of CTGF-induced EMT is mainly mediated via the TGF- $\beta$ signaling. Also, TGF- $\beta$ also affects the activity of many other pathways that can trigger EMT, such as Notch, Wnt, and integrin pathways. Based on the above facts, the amplification of FOXA1 and FOXA1-induced CTGF is expected to play a pivotal role in the progression of ovarian cancer by propelling the occurrence of EMT. To further examined the association between the development of EMT and FOXA1-CTGF- TGF- $\beta$ signaling pathway in the ovarian cancer cells, we studied the effects of lithium chloride, an inhibitor of TGF- $\beta$ pathway, on the cells [32].

\section{Materials And Methods}

\section{Data Collection and Analysis}

The expression and clinical data of The Cancer Genome Atlas (TCGA) and Genotype-Tissue Expression (GTEx) were downloaded from the UCSC Xena database (https://xenabrowser.net/datapages/). For FOXA1, the DNA copy number and methylation information were obtained from the cBioPortal database (https://www.cbioportal.org/). The survival analysis of FOXA1 in EOC database from GSE26193, GSE26712, and GSE63885 was performed using PrognoScan database.

\section{Gene Set Enrichment Analysis (GSEA)}

Correlation analyses between FOXA1 and other genes were performed using data from TCGA-OV cohort, and Pearson's correlation coefficient was calculated. GSEA was conducted using the R package "clusterProfiler" based on Gene Ontology (GO), Kyoto Encyclopedia of Genes and Genomes (KEGG), and Reactome pathway database.

\section{Correlation analysis of FOXA1 and drug response}

We downloaded half-inhibitory concentration (IC50) values of 192 anti-cancer drugs and FOXA1 expression profiles of 809 cell lines from the Genomics of Drug Sensitivity in Cancer database (GDSC: https://www.cancerrxgene.org/) and analyzed the Spearman's correlation between FOXA1 expression and IC50 values of anti-cancer drugs.

\section{Cell culture}

OVCAR3, A2780, 3AO, SKOV-3 (ovarian tumor cell lines) and IOSE80 (normal ovarian cell line) were purchased from ATCC of the United States. OVCAR3, 3AO and A2780 were cultured in RPMI 1640 medium supplemented with penicillin $(100 \mathrm{U} / \mathrm{mL})$, streptomycin $(100 \mathrm{mg} / \mathrm{L}), 10 \% \mathrm{v} / \mathrm{v}$ fetal bovine serum (FBS). IOSE80 and SKOV3 were cultured in DMEM and McCoy's 5A medium containing penicillin (100 $\mathrm{U} / \mathrm{mL})$, streptomycin $(100 \mathrm{mg} / \mathrm{L})$ and $10 \% \mathrm{FBS}$, respectively. The cells were cultured at $37{ }^{\circ} \mathrm{C}$ and $5 \%$ CO2. (The culture medium and supplements were purchased from Invitrogen)

\section{Silencing FOXA1 by using small interfering RNA (siRNA)}


FOXA1 siRNA and its negative control (NC) siRNA were purchased from Shanghai Sangon Co., Ltd. (Shanghai, China). Three siRNA sequences were as follows: siRNA\#1 (5'-GCGACUGGAACAGCUACUATT-3'; 5'-UAGUAGCUGUUCCAGUCGCTT-3'), SiRNA\#2 (5'-CCACUCGCUGUCCUUCAAUTT-3'; 5'AUUGAAGGACAGCGAGUGGTT-3', siRNA\#3 (5'-GCACUGCAAUACUCGCCUUTT-3'; 5'-

AAGGCGAGUAUUGCAGUGCTT-3'). Transfection of siRNA was performed by using Lipofectamine ${ }^{\text {TM }} 3000$ Transfection Reagent (Thermo Fisher). After transfection for $72 \mathrm{~h}$, the cells were collected to evaluate the knockdown efficiency of FOXA1 in OVCAR-3 cells $(n=6)$ by Western Blot and qPCR. The siRNA with the best efficiency was used in subsequent experiments.

\section{CCK-8 viability assay}

OVCAR3 cells transfected with siRNA-FoxA1 or siRNA-NC were cultured in a 96-well plate with $1 \times 10^{4}$ cells/well and 6 parallel wells in each group. The cells were cultured for 24,48 and $72 \mathrm{~h}$ respectively before CCK-8 assay. CCK-8 solution was incubated with cells at $37^{\circ} \mathrm{C}$ for $1-2 \mathrm{~h}$. The cell survival rate was detected at a wavelength of $450 \mathrm{~nm}$.

\section{Transwell assay}

Biocoat ${ }^{\mathrm{TM}}$ Matrigel ${ }^{\circledR}$ Invasion Chamber was rehydrated for $2 \mathrm{~h}$ at $37^{\circ} \mathrm{C}$ and $5 \% \mathrm{CO}_{2}$ using a filter with 8.0$\mu \mathrm{m}$ pore size (Corning, USA). After transfection with siRNA, the cells were collected and suspended ( $1 \times$ $10^{6} \mathrm{cells} / \mathrm{mL}$ ) in serum-free culture medium. $200 \mu \mathrm{L}$ of the cells in serum-free culture medium were added to the upper compartment, and $600 \mu \mathrm{L}$ RPMI 1640 containing 10\% FBS was added to the lower compartment. After $24 \mathrm{~h}$ of incubation at $37^{\circ} \mathrm{C}$ under $5 \% \mathrm{CO}_{2}$, the non-migrating cells were removed and culture medium was discarded. Filters were gently rinsed with PBS, and migrated cells were fixed with $4 \%$ $\mathrm{w} / \mathrm{v}$ formaldehyde for $15 \mathrm{~min} .0 .1 \%$ Crystal violet staining solution was used to stain the cells for $30 \mathrm{~min}$. The upper, middle and lower left and right fields were observed under the optical microscope (magnification $\times 100$ ) for cell counts for each assay. Cell migration was quantified with Image $\mathrm{J}$ software. Each group included three independently performed transwell assays.

\section{Immunofluorescence staining}

The immunofluorescence staining was performed on cells grown on $22 \times 22 \mathrm{~mm}$ coverslips. OVCAR-3 cells $\left(3 \times 10^{4} /\right.$ well) were grown in a 12 -well plate and cultured in the $37^{\circ} \mathrm{C}$ incubator overnight. At $72 \mathrm{~h}$ after the transfection with $80 \mathrm{nM}$ siRNA-FOXA1, $4 \% \mathrm{w} / \mathrm{v}$ paraformaldehyde was used for fixation for 30 min. $0.5 \% \mathrm{v} / \mathrm{v}$ Triton $\mathrm{X}-100$ was used in permeabilization for $5 \mathrm{~min}$ and $10 \%$ normal donkey serum for blocking for $1 \mathrm{~h}$ at room temperature. Then the cells were incubated at $4{ }^{\circ} \mathrm{C}$ overnight with the primary antibodies: mouse anti-E-cadherin (1:500, Abcam, USA) and rabbit anti-Vimentin (1:500, Abcam, USA). After washed by PBS for 3 times, secondary antibodies listed as follows were used for incubation for $1 \mathrm{~h}$ : Alexa Fluor® 488 Donkey Anti-mouse IgG or Anti-rabbit (1:200, Jackson ImmunoResearch, USA). Cells were finally counterstained with DAPI (Beyotime, China) for $5 \mathrm{~min}$ and the coverslips were mounted by 
using $10 \mu \mathrm{L}$ of FluroGuard anti-fade solution (Bio-Rad, USA). Images were taken using a confocal microscope (Lieca, Germany).

\section{Quantitative real-time PCR (qPCR)}

Cells or Tissues were collected and the RNAs were obtained by using TRIzol ${ }^{\circledR}$ Plus RNA Purification Kit (Thermo Fisher, Carlsbad, CA, USA) following the protocol. SuperScript ${ }^{\text {TM }}$ III First-Strand Synthesis SuperMix for qRT-PCR囚Thermo Fisher, Carlsbad, CA, USA『was used to synthesize cDNA. Real-time PCR was carried out by applying PowerU ${ }^{\mathrm{TM}} \mathrm{SYBR}^{\mathrm{TM}}$ Green Master Mix (Applied Biosystems, Carlsbad, CA, USA). The cycling program was set as: $95^{\circ} \mathrm{C}, 2 \mathrm{~min} ; 40$ cycles of amplification $\left(95^{\circ} \mathrm{C}, 15 \mathrm{~s} ; 60^{\circ} \mathrm{C}, 1 \mathrm{~min}\right)$. The sequences of the primers used in this experiment were as follows: FOXA1, 5'-GCATACGAA CAGGCACTGCAATACT-3区(forward) and 5'-GTGTTTAGGACGGGTCTGGAATA-3区(reverse); GAPDH: 5'CCATGACAACTTTGGTATCGTGGAA -3囚(forward) and 5'-GGCCATCACGCCACAGTTTC-3囚(reverse). GAPDH was used as internal control for normalization. The relative expression of the target genes was evaluated by the $2^{-\Delta \Delta \mathrm{Ct}}$ method.

\section{Western blot}

Total cytoplasmic and nuclear proteins from OVCAR-3 cells were extracted with RIPA Buffer (Thermo Fisher, USA). NE-PERTM Nuclear and Cytoplasmic Extraction Kit (Thermo Fisher, USA) added with Protease and Phosphatase Inhibitor Cocktail (Thermo Fisher, USA). BCA protein assay kit (Beyotime Biotechnology, China) was used for quantification. Protein $(30 \mu \mathrm{g})$ was loaded onto SDS-PAGE gel, and transferred to a Hybond-P PVDF membrane (GE Healthcare, USA). 5\% fat-free milk in TBST (Tris-buffered saline with $0.1 \%$ Tween-20) was used for blocking. Following antibodies were incubated with the membranes at $4^{\circ} \mathrm{C}$ overnight: mouse anti-E-cadherin (1:1000, Abcam, USA), rabbit anti-Vimentin (1:1000, Abcam, USA), rabbit anti-Snail (1:1000, Abcam, USA), and GAPDH (1:5000, Abcam, USA) as an internal control. Protein expression was visualized on X-ray films using the HRP-conjugated goat anti- mouse or rabbit secondary antibodies (1:5000, Thermo Fisher, USA) and SuperSignal West Dura Extended Duration Substrate (Thermo Fisher, USA). Band intensities were quantitated using Image Pro Plus 6.0 software. The results were presented as the density ratio of the target protein band to the internal control.

\section{Construction of the CTGF promoter luciferase reporter plasmids}

The binding site of FOXA1 with CTGF was predicted on LASAGNA-search. Sequences of the wild type CTGF promoter (-500 to -1$)$ and mutant CTGF promoter (-399 to -390 , CAGGGCAAAC to CACCGCTTAC) with recognition sites specific for the enzymes Kpnl/Xhol were manufactured by Sangon Biotech (Shanghai). FOXA1 CDS flanked with BamHI/EcoRI was cloned as well. Luciferase reporter plasmids, pGL3-Basic-CTGF-w (wild type) and pGL3-Basic-CTGF-m (mutant), were constructed by having the primers flanked with $\mathrm{Kpnl} /$ Xho cloned into the $\mathrm{Kpnl} /$ Xho sites of $\mathrm{pGL3-Basic} \mathrm{vector} \mathrm{(Promega).} \mathrm{The}$ FOXA1 overexpression plasmid was created by cloning the FOXA1 CDS sequence with BamHI/EcoRI into pcDNA3.1 (Invitrogen). pRL-TK vector (Promega) \the renilla luciferase plasmid, was adopted as an internal control reporter vector. 


\section{Dual-luciferase reporter assays}

OVCAR3 cells were co-transfected with a combination of plasmids comprising of either a mutant or wildtype CTGF reporter gene plasmid, a pRL-TK Renilla luciferase reporter plasmid, and a FOXA1 overexpression plasmid. Thus, the cells were transfected with the combination of plasmids as following respectively: pGL3-Basic/pcDNA3.1-FOXA1/ pRL-TK; or pGL3-Basic-CTGF-w/pcDNA3.1-FOXA1/pRL-TK; or pGL3-Basic-CTGF-m/pcDNA3.1-FOXA1/pRL-TK group. Activities of firefly renilla luciferases were measured $48 \mathrm{~h}$ after transfection according to the dual luciferase reporter assay system, with six replicas in each group (Promega).

\section{Effects of FOXA1 silencing on CTGF/TGF- $\beta$ pathway and EMT-associated markers}

OVCAR3 cells were transfected with siRNA-NC or siRNA-FOXA1, which was followed by replacing with the serum-free culture medium after $12 \mathrm{~h}$. The TGF- $\beta 1$ groups were stimulated with $10 \mathrm{ng} / \mathrm{ml}$ TGF- $\beta 1$ accordingly (R\&D systems). Cells were collected after $48 \mathrm{~h}$ for Western Blot analysis for FOXA1, CTGF, MMP-2, E-cadherin and snail using rabbit-anti-CTGF antibody (1:1000, Abcam, USA), and rabbit-antiMMP2 antibody (1: 500, Abcam, USA).

\section{Lithium chloride treatment}

The cells were grouped as follows: control; siRNA-NC-transfected; siRNA-NC-transfected and LiCl treatment; siRNA-FOXA1-transfected; siRNA-FOXA1-transfected and LiCl treatment. A final concentration of $10 \mathrm{mM} \mathrm{LiCl}$ was added to cells after transfection of siRNA-NC or siRNA-FOXA1 for $8 \mathrm{~h}$.

\section{Statistical analysis}

SPSS 17.0 software (SPSS Inc., Chicago, IL, USA) was used for statistical analysis. The results were expressed as average value \pm standard deviation (SD). The paired, two-tailed Student's t-test was used to compare the results between two groups. Two-sided $p$ value less than 0.05 was regarded as statistically significant. ${ }^{*} p<0.05 ;{ }^{* \star} p<0.01 ; * \star \star p p<0.001$; and ${ }^{\star \star \star *} p<0.0001$.

\section{Results}

\section{Pan-cancer expression of FOXA1}

We first evaluate the pan-cancer expression of FOXA1. Results revealed that FOXA1 was highly expressed in 19 tumor types, including BRCA, CESC, COAD, DLBC, ESCA, KIRC, LGG, LIHC, LUAD, LUSC, OV, PAAD, PRAD, READ, STAD, THCA, THYM, UCEC, and UCS. In comparison, low FOXA1 expression was observed in ACC, GBM, HNSC, KICH, LAML, SKCM, and TGCT (Fig. 1A). In paired tumor and adjacent normal tissues, FOXA1 was over-expressed in BLCA, BRCA, CESC, LUAD, PAAD, PRAD, and STAD, while low-expressed in COAD, HNSC, KICH, KIRC, and READ (Fig. 1B). In addition, we also observed that FOXA1 expression was higher in worse tumor stages in ACC, KIRC, KIRP, BRCA, and THCA (Fig. 2A-E), while lower in ESCA, BLCA, COAD, and READ (Fig. 2F-I). 
Genetic and epigenetic alterations induce changes in gene expression. We explored genetic alterations in FOXA1 using cBioPortal and observed that patients in prostate adenocarcinoma and non-small cell lung cancer has high genetic alterations of FOXA1 (Fig. 3A). The copy number values were positively correlated with FOXA1 expression and the methylation levels of the FOXA1 promoter were negatively correlated with FOXA1 expression (Fig. 3B-C).

\section{The prognostic value of FOXA1}

To assess the prognostic value of FOXA1, we performed Kaplan-Meier survival analysis in pan-cancer. Kaplan-Meier survival analysis revealed that high FOXA1 expression predicted worse overall survival of patients with ACC, BRCA, KIRC, KIRP, LGG, MESO, SARC, SKCM, and THCA (Fig. 4A-I), while better survival of patients with BLCA, COAD, and PAAD (Fig. 4J-L). For data of EOC from GEO database, we found that high FOXA1 predicted poorer survival status of EOC patients in GSE26193, GSE26712, and GSE63885 (Fig. 5A-C). To explore the association between FOXA1 expression and TME, we downloaded signature gene sets of TME from published articles and calculated signature scores according to the method described previously [33]. Results revealed that EMT related pathways was positively correlated with FOXA1 in pan-cancer.

\section{Expressions of FOXA1 in EOC cell lines}

The RNA materials from the cancer and normal cells lines were extracted for reverse transcription and RTPCR assay (Fig. 6A). The relative mRNA level of FOXA1 is significantly upregulated in the OVCAR3 cell line compared to the rest. Western blot found that the protein level of FOXA1 was significantly upregulated in ovarian cancer cells compared with the normal cell line IOSE80 (Fig. 6B-C). Among all the ovarian cancer cell lines tested (i.e. OVCAR-3/A2780/3AO/SKOV-3), both transcriptional and translational levels of FOXA1 were highest in OVCAR3, followed by $3 A O$ and SKOV-3, and then A2780.

\section{F0XA1 expression was significantly inhibited by siRNA silencing}

Three siRNAs, siRNA-1, siRNA-2 and siRNA-3, specific for different sites of FOXA1 gene were designed for silencing FOXA1 in OVCAR3 by transient transfection. At the same time, the potency of the three siRNA was compared. The mRNA and protein expressions of FOXA1 were remarkably suppressed with siRNA-2 transfection compared to the other groups (Fig. 6D-F). Thus, siRNA-2 was employed as the most effective interference siRNA in the following experiments.

\section{FOXA1 silencing markedly attenuated cell proliferation and invasion}

The proliferative and invasive capabilities of FOXA1 knockdown cells were examined by the CCK8 and transwell assays respectively. The proliferation of cells was significantly suppressed following the transfection of siRNA-FOXA1 compared with siRNA-NC (Fig. 7A). The results from transwell assay 
indicated that the invasiveness was also significantly inhibited due to the absence of FOXA1 in siRNAFOXA1 OVCAR-3 cells (Fig. 7B-C).

\section{FOXA1 silencing inhibited EMT}

OVCAR3 cells were transfected with siRNA-FOXA1 and siRNA-NC respectively. EMT-associated markers in OVCAR3 cells were detected by Western blot and immunofluorescence staining. As shown in Fig. 7D-G, the results indicated that FOXA1 silencing inhibited EMT as shown by the remarkably increased expression of E-cadherin and decreased levels of Vimentin $(p<0.01)$. It was indicated that FOXA1 might serve as an activator in OVCAR3 cells via promoting EMT.

\section{FOXA1 regulated CTGF expression by binding to its promoter region}

To explore the relation between FOXA1 and CTGF, OVCAR3 were transfected with either a wild-type CTGF promoter or a mutant CTGF promoter, the predicted binding site and the mutated sequence were defined as in Fig. 8A. The results from dual-luciferase reporter assays showed that, comparing to the pGL3-Basic control group, the activity of pGL3-Basic-CTGF-w luciferase was significantly enhanced $(p<0.01)$; while the activity of pGL3-Basic-CTGF-m was higher than that of pGL3-Basic-CTGF-w was significantly downregulated $(p<0.01)$ (Fig. 8B). It suggests that FOXA1 initiates transcription of CTGF by binding to its promoter.

\section{FOXA1-mediated EMT was dependent on the activation of CTGF/TGF- $\beta$ pathway}

In order to confirm that the molecular mechanism of FOXA1-induced EMT relies on activation of the CTGF/TGF- $\beta$ signaling pathway, FOXA1 of OVCAR-3 cells was silenced followed by a stimulation with TGF- $\beta 1$ in vitro. Western blot analysis showed that both CTGF and FOXA1 expressions were considerably elevated in the TGF- $\beta 1$ stimulated groups (siRNA-NC + TGF- $\beta 1$ and siRNA-FOXA1 + TGF- $\beta 1$ ) compared to the non-stimulated groups (siRNA-NC and siRNA-FOXA1) (Fig. 8C). When comparing the groups siRNA-NC vs. siRNA-FOXA1 as well as siRNA-NC + TGF- $\beta 1$ vs. siRNA-FOXA1 + TGF- $\beta 1$, it is found that the expressions of CTGF and FOXA1 were significantly suppressed due to FOXA1 ablation. In addition, the EMT-associated proteins, MMP2 and snail were significantly upregulated following the treatment of exogenous TGF- $\beta 1(p<0.01)$, whereas their expressions were considerably reduced $(p<0.05, p<0.01)$ following FOXA1 ablation. Congruently, the cell-cell junction indicator protein, E-cadherin, showed a prominent upregulation in FOXA1-silenced cells $(p<0.01)$, and a downregulation in TGF- $\beta 1$ stimulated cells $(p<0.01)$. Here, it shows that interference with FOXA1 down-regulates the expression of CTGF and thus inhibiting the activation of CTGF/TGF- $\beta$ pathway in OVCAR3 cells, which in turn attenuates the occurrence and development of EMT that is mediated by the TGF- $\beta$ signaling pathway.

\section{The inhibitory effect of LiCl on FOXA1 and EMT in OVCAR3 cells}


To examine the inhibitory effects of $\mathrm{LiCl}$ on the FOXA1-CTGF-TGF- $\beta$ pathway, OVCAR3 cells were treated accordingly as per the description of the groups, namely the siRNA-FOXA1 + $10 \mathrm{mM}$ LiCl, siRNA-NC + 10 mM LiCl, siRNA-FOXA1, and siRNA-NC groups. The protein expression levels of FOXA1, vimentin, Ecadherin and snail in the treated cells were detected by western blot assays. In the single treatment group with either LiCl (siRNA-NC + 10mM LiCl) or FOXA1 knockdown (siRNA-FOXA1), expressions of FOXA1, CTGF, cleaved-TGF-beta and EMT-associated markers were all downregulated (Fig. 8D). The potency of suppression was strongest in the combination treatment group (siRNA-FOXA1 + LiCl). The combination treatment of FOXA1 knockdown and administration of lithium chloride exerts a more robust inhibitory effect on the EMT-associated proteins, compared to the respective single treatment. The epithelial marker, E-cadherin, was upregulated in the treatment groups, indicating a recovery of the cell-cell adhesive junctions. Our data show that the LiCl treatment is able to suppress the FOXA1-CTGF-TGF- $\beta$ pathway and, therefore, inhibit EMT in OVCAR3 cells. Equally important, silenced FOXA1 and LiCl treatment could have worked collaboratively to augment the inhibitory impact on EMT features.

\section{The correlation between FOXA1 and immunosuppressive microenvironment}

We further performed the GSEA of FOXA1 in TCGA-OV cohort based on GO, KEGG, and Reactome database. Results revealed immune-related pathways were commonly enriched (Fig. 9A-C). Through the correlation analyses between FOXA1 and MHC genes (Fig. 10A), immunosuppressive genes (Fig. 10B), immune activating genes (Fig. 10C), and chemokine receptors (Fig. 10D), we found that FOXA1 was closely correlated with immune regulatory genes, indicating a pivotal role of FOXA1 in tumor immunomodulatory function, especially in TCGA-OV cohort.

\section{The correlation between FOXA1 and resistant of anti-cancer drugs}

At last, we conducted the correlation analyses between FOXA1 expression and IC50 values of anti-cancer drugs. We found that the expression of FOXA1 positively correlated IC50 values of most anti-cancer drugs, such as Camptothecin, Vinblastine, and Cisplatin (Fig. 11, Table 1). These results indicated that EOC patients with high expression of FOXA1 may be resistant to most anti-cancer drugs treatments.

\section{Discussion}

In our research, we investigated the potential role of the FOXA1, a transcription factor, in pan-cancer and o EOC and its underlying molecular mechanism on promoting EMT. In pan-cancer research, we found that FOXA1 was highly expressed in most tumor types. High expression of FOXA1 predicted poorer survival of patients with EOC. In the experimental verification, FOXA1 expression levels in four ovarian cancer cell lines were all higher than those in normal ovarian cells. The overexpression was most prominent in OVCAR-3 with the most malignant characteristics, implying that FOXA1 had an evitable role in the development of EOC. Preliminary studies demonstrated that FOXA1 silencing could effectively hinder the invasion and proliferation of certain tumor cells, for example, lung adenocarcinoma A549 cells [7] and 
lung squamous cell carcinoma cells [34]. These results were consistent with what we found in our research. In FOXA1 knockdown OVCAR3 cells, the mesenchymal characteristics were reversed, which is confirmed by the upregulated E-cadherin level and downregulated snail and vimentin.

It was demonstrated that FOXA1 have a directly and/or indirectly role in the regulation of EMT occurrence. Our findings were consistent with the results in other research. For example, Badve et al. implied that the concentration of FOXA1 varied at different stages in the course of tumor progression with a significant correlation with drug resistance and poor prognosis in both breast and prostate tumors [25]. In addition, FOXA1 was upregulated in malignant ovarian cancer tissues with substantial differences between the early and advanced stages [21]. On the other hand, researchers also pointed out that FOXA1 might suppress tumorigenesis in some cancer types by inhibiting EMT. For example, Song Y et al. suggested that the inhibition of FOXA1 expression could provoke the activation of EMT in pancreatic cancer [35]. Song $L$ et al. found that a high level of FOXA1 inhibited cell invasion and proliferation in breast cancer [35]. In another breast cancer research, FOXA1 was found to downregulate EMT-associated markers, including E-cadherin, ZEB2 and vimentin, eventually preventing EMT progression [37]. Likewise, similar outcomes were also observed in liver cancer, nasopharyngeal cancer, gastric cancer, prostate cancer and colorectal cancer, in which the EMT process seemed to be reversed due to the presence of FOXA1 [38]. The possible explanation could be that FOXA1, as a strong activator of E-cadherin transcription, could serve as a tumor suppressor gene which could possibly reverse EMT by increasing Ecadherin expression, restoring the epithelial phenotype of the cancer cells [35]. Taken together, FOXA1 might influence the viability, proliferation and invasion of tumor cells by affecting different signaling pathways in different cancers, leading to various effects on EMT.

Activated CTGF/TGF- $\beta$ pathway leads to a loss of adhesion between cells, accelerating the development of EMT and metastasis in tumours[38]. There are many researches on CTGF pointing out its direct or indirect role in facilitating tissue fibrosis or profibrotic TGF- $\beta 1$ activity [32, 34-36]. Persistent activation of TGF- $\beta$ pathway is associated with malignancies of cancers. Nguyen and Goldschmeding have comprehensively explained the mechanisms of CTGF in potentiating and enhancing TGF- $\beta$ signaling either by increasing the affinity between TGF- $\beta$ molecule and its receptor through physical interactions in the extracellular matrix, or by abrogating the negative TGF- $\beta$ feedback loop (Smad7) following binding to TrkA [39]. The present study proves the interaction between FOXA1 and the promoter of CTGF, suggesting that FOXA1 can increase the transcription of CTGF. Transcribed CTGF entering the ECM functions as a strong enhancing mediator of the TGF- $\beta$ signaling pathway. This helps to explain the reason why TGF- $\beta$ signaling was significantly enhanced in OVCAR3 cells, especially in those stimulated with the TGF- $\beta$ cytokine. Furthermore, it has been shown that the persistence and severity of fibrosis caused by simultaneous injection of both TGF- $\beta$ and CTGF far more exceeds that caused by the injection of individual TGF- $\beta$ or CTGF on its own. In the study of renal interstitial fibrosis, CTGF is an important cytokine that affect the prognosis and progression of disease [40]. Congruently, our study found that FOXA1, CTGF and TGF- $\beta$ are intercorrelated. Overexpression of FOXA1 is highly correlated with the elevation of endogenous CTGF and cleaved-TGF- $\beta$ in OVCAR3. This is because that in cancer cells, amplified FOXAl upregulates the expression of CTGF, which elicits prolonged activation of the CTGF/TGF- 
$\beta$ pathway. Thereby, many important TGF- $\beta$-pathway-induced elements associated with EMT features, cell proliferation and invasion, and ECM remodeling are expected to be modulated correspondingly.

$\mathrm{LiCl}$, as an inhibitor for GSK-3, has been reported to inhibit EMT efficiently[41]. In accordance with the previous findings, our study showed that $\mathrm{LiCl}$ leads to downregulations of FOXA1, CTGF and cleavedTGF- $\beta$ in OVCAR3 cells, indicating the potential role of $\mathrm{LiCl}$ in controlling EMT progression in EOC. Further analysis demonstrated that $\mathrm{LiCl}$ combined with siRNA-FOXA1 silencing exerted a more robust effect on inhibiting EMT in OVCAR3.

Tumor microenvironment, especially tumor immune microenvironment, play a vital role in accelerating tumor progression. In our study, we predicted that FOXA1 is involved in immune regulation-related pathways using GSEA. Moreover, FOXA1 was positively correlated with MHC genes, immunosuppressive genes, immune activating genes, and chemokine receptors, indicating a pivotal role of FOXA1 in tumor immunomodulatory function, especially in TCGA-OV cohort. Further analysis indicated that high expression of FOXA1 may be resistant to most anti-cancer drugs treatments, such as Camptothecin, Vinblastine, and Cisplatin.

In conclusion, we conducted a comprehensive assessment of FOXA1, revealing a potential role of FOXA1 as an indicator of patient prognosis and molecular mechanism of FOXA1 to promote EMT in ovarian cancer. We also predicted that FOXA1 was involved in immune regulation-related pathways. In addition, EOC patients with high FOXA1 expression may be resistant to most anti-cancer drugs treatments.

\section{Abbreviations}

EMT, epithelial-mesenchymal transition; EOC, Epithelial ovarian cancer; LiCl, lithium chloride; MMPs, matrix metallopeptidases; bHLH, basic helix-loop-helix; FOXA1, forkhead-box A1; OS, overall survival; FBS, fetal bovine serum; DMEM, Dulbecco's modified Eagle's medium; ANOVA analysis of variance; TCGA, The Cancer Genome Atlas; ACC, Adrenocortical carcinoma; BLCA, Bladder urothelial carcinoma; BRCA, Breast invasive carcinoma; COAD, Colon adenocarcinoma; DLBC, Diffuse large B-cell lymphoma; ESCA, Esophageal carcinoma; KICH, Kidney chromophobe; KIRC, Kidney renal clear cell carcinoma; LGG, Lowgrade glioma; LIHC, Liver hepatocellular carcinoma; LUAD, Lung adenocarcinoma; LUSC, Lung squamous cell carcinoma; PFI, Progression-free interval; PRAD, Prostate adenocarcinoma; READ, Rectum adenocarcinoma; SKCM, Skin cutaneous melanoma; STAD, Stomach adenocarcinoma; TGCT, Testicular germ cell tumors; THCA, Thyroid carcinoma; UCEC, Uterine corpus endometrial carcinoma; UCS, Uterine carcinosarcoma

\section{Declarations}

\section{Acknowledgments}

We thank Dr.Liang Xia for providing the database information and bioinformatic analysis. 
Author contributions

K.W., X.F., and L.Z. conceptualization; K.W., J.Y., C.G., and J.Y. investigation; X.S., S.M., X.C., and L.Z. supervision; K.W. and X.S. methodology; X.C., and L.Z. funding acquisition; S.M. validation; K.W. and L.Z. writing original draft.

\section{Funding and additional information}

The present study was supported by the Science and Technology Bureau of Zhejiang Province (Grant No. LGF19H160019), Taizhou Science and Technology Project, Zhejiang Province (1802KY10), The Scientific Innovation Foundation of Taizhou Hospital of Zhejiang Province (18EZZDC7囚15EZD15).

\section{Conflict of interest}

The authors declare that they have no conflicts of interest with the contents of this article.

Ethics approval and consent to participate

Informed consent was obtained from all individual participants for whom identifying information was included in this article.

\section{Consent for publication}

All the authors report no disclosures relevant to the manuscript.

\section{Data availability statements}

The datasets generated and/or analyzed during the current study are available from the corresponding author upon reasonable request.

\section{References}

1. Poveda A: Ovarian cancer treatment: what is new. INT J GYNECOL CANCER 2003, 13 Suppl 2:241250.

2. Pautier $P$, Gutierrez-Bonnaire M, Rey A, Sillet-Bach I, Chevreau C, Kerbrat P, Morice P, Duvillard $P$, Lhommé C: Combination of bleomycin, etoposide, and cisplatin for the treatment of advanced ovarian granulosa cell tumors. INT J GYNECOL CANCER 2008, 18(3):446-452.

3. Zhao D, Zhang W, Li XG, Wang XB, Li M, Li YF, Tian HM, Song PP, Liu J, Chang QY et al: [The mRNA expression of BRCA1, ERCC1, TUBB3, PRR13 genes and their relationship with clinical chemosensitivity in primary epithelial ovarian cancer]. Zhonghua Zhong Liu Za Zhi 2012, 34(3):196200.

4. Skirnisdottir I, Seidal T: Association of p21, p21 p27 and p21 p53 status to histological subtypes and prognosis in low-stage epithelial ovarian cancer. Cancer Genomics Proteomics 2013, 10(1):27-34. 
5. van der Burg ME, van Lent M, Buyse M, Kobierska A, Colombo N, Favalli G, Lacave AJ, Nardi M, Renard J, Pecorelli S: The effect of debulking surgery after induction chemotherapy on the prognosis in advanced epithelial ovarian cancer. Gynecological Cancer Cooperative Group of the European Organization for Research and Treatment of Cancer. N Engl J Med 1995, 332(10):629-634.

6. Yin M, Li C, Li X, Lou G, Miao B, Liu X, Meng F, Zhang H, Chen X, Sun M et al: Over-expression of LAPTM4B is associated with poor prognosis and chemotherapy resistance in stages III and IV epithelial ovarian cancer. J SURG ONCOL 2011, 104(1):29-36.

7. Li J, Zhang S, Zhu L, Ma S: Role of transcription factor FOXA1 in non-small cell lung cancer. $M O L$ MED REP 2018, 17(1):509-521.

8. Valastyan S, Weinberg RA: Tumor metastasis: molecular insights and evolving paradigms. $C E L L$ 2011, 147(2):275-292.

9. Mendez MG, Kojima S, Goldman RD: Vimentin induces changes in cell shape, motility, and adhesion during the epithelial to mesenchymal transition. FASEB J 2010, 24(6):1838-1851.

10. Ribatti D, Tamma R, Annese T: Epithelial-Mesenchymal Transition in Cancer: A Historical Overview. TRANSL ONCOL 2020, 13(6):100773.

11. Ruscetti M, Quach B, Dadashian EL, Mulholland DJ, Wu H: Tracking and Functional Characterization of Epithelial-Mesenchymal Transition and Mesenchymal Tumor Cells during Prostate Cancer Metastasis. CANCER RES 2015, 75(13):2749-2759.

12. Ye LC, Liu TS, Ren L, Wei Y, Zhu DX, Zai SY, Ye QH, Yu Y, Xu B, Qin XY et al: Randomized controlled trial of cetuximab plus chemotherapy for patients with KRAS wild-type unresectable colorectal liverlimited metastases. J CLIN ONCOL 2013, 31(16):1931-1938.

13. Gros J, Tabin CJ: Vertebrate limb bud formation is initiated by localized epithelial-to-mesenchymal transition. SCIENCE 2014, 343(6176):1253-1256.

14. Yoshino M, Tsutsumi K, Kanazawa A: Profiles of embryonic nuclear protein binding to the proximal promoter region of the soybean $\beta$-conglycinin a subunit gene. Plant Biol (Stuttg) 2015, 17(1):147152.

15. Asthana S, Goyal P, Dhar R, K U, Pampanaboina NB, Christakiran J, Sagiri SS, Khanna M, Samal A, Banerjee I et al: Evaluation extracellular matrix-chitosan composite films for wound healing application. J Mater Sci Mater Med 2015, 26(8):220.

16. Grande MT, Sánchez-Laorden B, López-Blau C, De Frutos CA, Boutet A, Arévalo M, Rowe RG, Weiss SJ, López-Novoa JM, Nieto MA: Snail1-induced partial epithelial-to-mesenchymal transition drives renal fibrosis in mice and can be targeted to reverse established disease. NAT MED 2015, 21(9):989997.

17. Lovisa S, LeBleu VS, Tampe B, Sugimoto H, Vadnagara K, Carstens JL, Wu CC, Hagos Y, Burckhardt BC, Pentcheva-Hoang $\mathrm{T}$ et al: Epithelial-to-mesenchymal transition induces cell cycle arrest and parenchymal damage in renal fibrosis. NAT MED 2015, 21(9):998-1009.

18. Khodzhigorova A, Distler A, Lang V, Dees C, Schneider H, Beyer C, Gelse K, Distler O, Schett G, Distler $\mathrm{JH}$ : Inhibition of sumoylation prevents experimental fibrosis. ANN RHEUM DIS 2012, 71(11):1904- 
1908.

19. Correa-Costa M, Braga TT, Felizardo RJ, Andrade-Oliveira V, Perez KR, Cuccovia IM, Hiyane MI, Da SJ, Câmara NO: Macrophage trafficking as key mediator of adenine-induced kidney injury. Mediators Inflamm 2014, 2014:291024.

20. Teeuwssen M, Fodde R: Wnt Signaling in Ovarian Cancer Stemness, EMT, and Therapy Resistance. $J$ CLIN MED 2019, 8(10).

21. Wang K, Guan C, Fang C, Jin X, Yu J, Zhang Y, Zheng L: Clinical significance and prognostic value of Forkhead box A1 expression in human epithelial ovarian cancer. ONCOL LETT 2018, 15(4):44574462.

22. Williamson EA, Wolf I, O'Kelly J, Bose S, Tanosaki S, Koeffler HP: BRCA1 and F0XA1 proteins coregulate the expression of the cell cycledependent kinase inhibitor p27(Kip1). ONCOGENE 2006, 25(9):1391-1399.

23. Habashy HO, Powe DG, Rakha EA, Ball G, Paish C, Gee J, Nicholson RI, Ellis IO: Forkhead-box A1 (FOXA1) expression in breast cancer and its prognostic significance. EUR J CANCER 2008, 44(11):1541-1551.

24. Nucera C, Eeckhoute J, Finn S, Carroll JS, Ligon AH, Priolo C, Fadda G, Toner M, Sheils O, Attard M et al: FOXA1 is a potential oncogene in anaplastic thyroid carcinoma. CLIN CANCER RES 2009, 15(11):3680-3689.

25. Badve S, Turbin D, Thorat MA, Morimiya A, Nielsen TO, Perou CM, Dunn S, Huntsman DG, Nakshatri $\mathrm{H}$ : FOXA1 expression in breast cancer-correlation with luminal subtype A and survival. CLIN CANCER RES 2007, 13(15 Pt 1):4415-4421.

26. Lin L, Miller CT, Contreras JI, Prescott MS, Dagenais SL, Wu R, Yee J, Orringer MB, Misek DE, Hanash SM et al: The hepatocyte nuclear factor 3 alpha gene, HNF3alpha (FOXA1), on chromosome band $14 q 13$ is amplified and overexpressed in esophageal and lung adenocarcinomas. CANCER RES 2002, 62(18):5273-5279.

27. Lin M, Pan J, Chen Q, Xu Z, Lin X, Shi C: Overexpression of FOXA1 inhibits cell proliferation and EMT of human gastric cancer AGS cells. GENE 2018, 642:145-151.

28. Gerhardt J, Montani M, Wild P, Beer M, Huber F, Hermanns T, Müntener M, Kristiansen G: FOXA1 promotes tumor progression in prostate cancer and represents a novel hallmark of castrationresistant prostate cancer. AM J PATHOL 2012, 180(2):848-861.

29. Strazzabosco M: Foxa1 and Foxa2 regulate bile duct development in mice. J HEPATOL 2010, 52(5):765-767.

30. Deng YZ, Chen PP, Wang Y, Yin D, Koeffler HP, Li B, Tong XJ, Xie D: Connective tissue growth factor is overexpressed in esophageal squamous cell carcinoma and promotes tumorigenicity through betacatenin-T-cell factor/Lef signaling. J BIOL CHEM 2007, 282(50):36571-36581.

31. Sonnylal S, Xu S, Jones H, Tam A, Sreeram VR, Ponticos M, Norman J, Agrawal P, Abraham D, de Crombrugghe $\mathrm{B}$ : Connective tissue growth factor causes EMT-like cell fate changes in vivo and in vitro. J CELL SCI 2013, 126(Pt 10):2164-2175. 
32. Choi SI, Kim BY, Dadakhujaev S, Jester JV, Ryu H, Kim TI, Kim EK: Inhibition of TGFBlp expression by lithium: implications for TGFBI-linked corneal dystrophy therapy. Invest Ophthalmol Vis Sci 2011, 52(6):3293-3300.

33. Mariathasan S, Turley SJ, Nickles D, Castiglioni A, Yuen K, Wang Y, Kadel El, Koeppen H, Astarita JL, Cubas $\mathrm{R}$ et al: TGF $\beta$ attenuates tumour response to PD-L1 blockade by contributing to exclusion of T cells. NATURE 2018, 554(7693):544-548.

34. Song Y, Washington MK, Crawford HC: Loss of FOXA1/2 is essential for the epithelial-tomesenchymal transition in pancreatic cancer. CANCER RES 2010, 70(5):2115-2125.

35. Song $L$, Xu Z, Li L, Hu M, Cheng L, Chen L, Zhang B: Forkhead box protein A1 inhibits the expression of uncoupling protein 2 in hydrogen peroxide-induced A549 cell line. Cell Stress Chaperones 2014, 19(1):53-60.

36. Zhang XL, Wang HS, Liu N, Ge LC: Bisphenol A stimulates the epithelial mesenchymal transition of estrogen negative breast cancer cells via FOXA1 signals. ARCH BIOCHEM BIOPHYS 2015, 585:10-16.

37. Winkler J, Abisoye-Ogunniyan A, Metcalf $\mathrm{KJ}$, Werb Z: Concepts of extracellular matrix remodelling in tumour progression and metastasis. NAT COMMUN 2020, 11(1):5120.

38. Nguyen TQ, Goldschmeding R: Bone morphogenetic protein-7 and connective tissue growth factor: novel targets for treatment of renal fibrosis? Pharm Res 2008, 25(10):2416-2426.

39. Okada H, Kikuta T, Kobayashi T, Inoue T, Kanno Y, Takigawa M, Sugaya T, Kopp JB, Suzuki H: Connective tissue growth factor expressed in tubular epithelium plays a pivotal role in renal fibrogenesis. J AM SOC NEPHROL 2005, 16(1):133-143.

40. Burns WC, Twigg SM, Forbes JM, Pete J, Tikellis C, Thallas-Bonke V, Thomas MC, Cooper ME, Kantharidis $\mathrm{P}$ : Connective tissue growth factor plays an important role in advanced glycation end product-induced tubular epithelial-to-mesenchymal transition: implications for diabetic renal disease. J AM SOC NEPHROL 2006, 17(9):2484-2494.

41. Novetsky AP, Thompson DM, Zighelboim I, Thaker PH, Powell MA, Mutch DG, Goodfellow PJ: Lithium chloride and inhibition of glycogen synthase kinase $3 \beta$ as a potential therapy for serous ovarian cancer. INT J GYNECOL CANCER 2013, 23(2):361-366.

\section{Tables}

Table file is not available with this version.

\section{Figures}



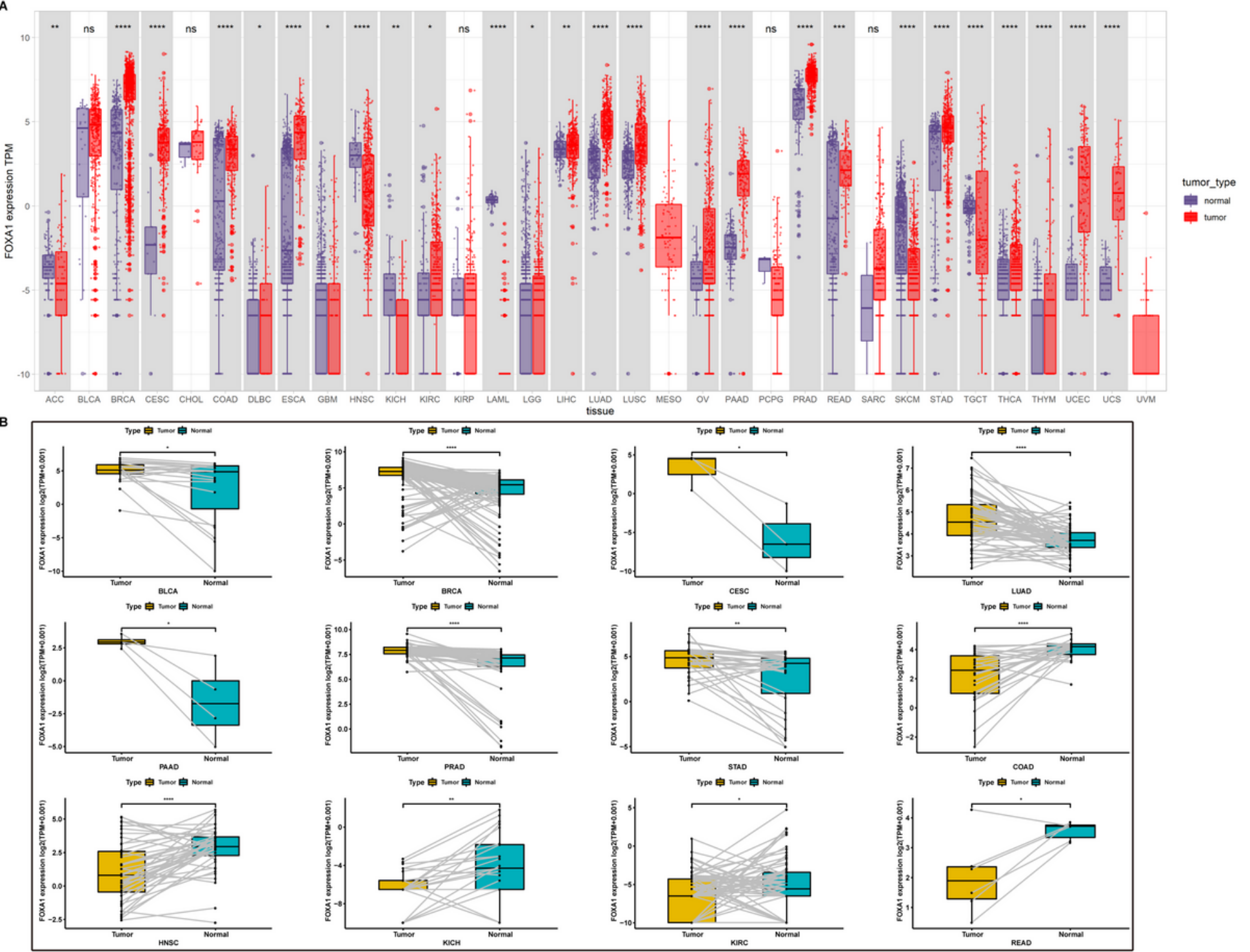

Figure 1

Pan-cancer expression. (A) Pan-cancer expression of FOXA1. (B) The expression of FOXA1 in paired tumor and adjacent normal tissues in indicated tumor types from TCGA cohort. 
A
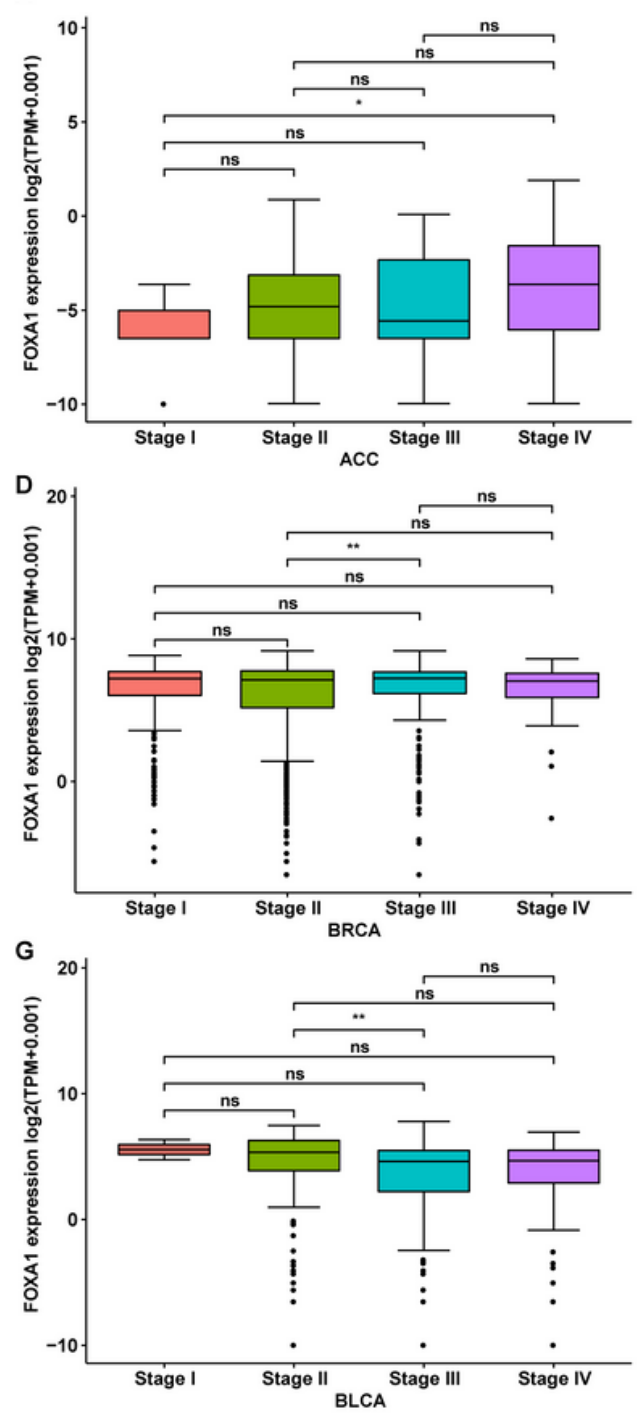

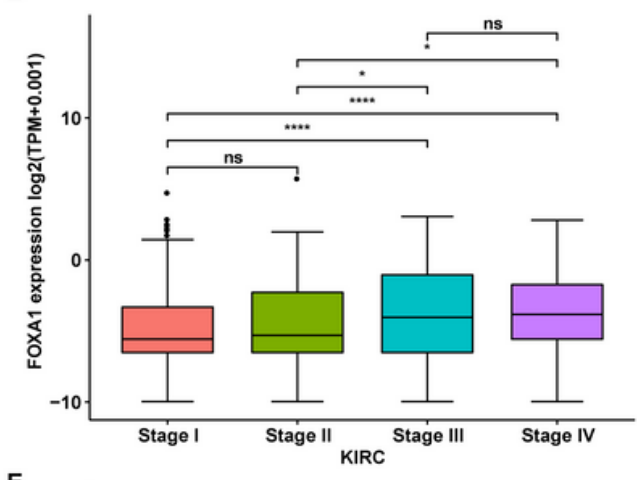

E
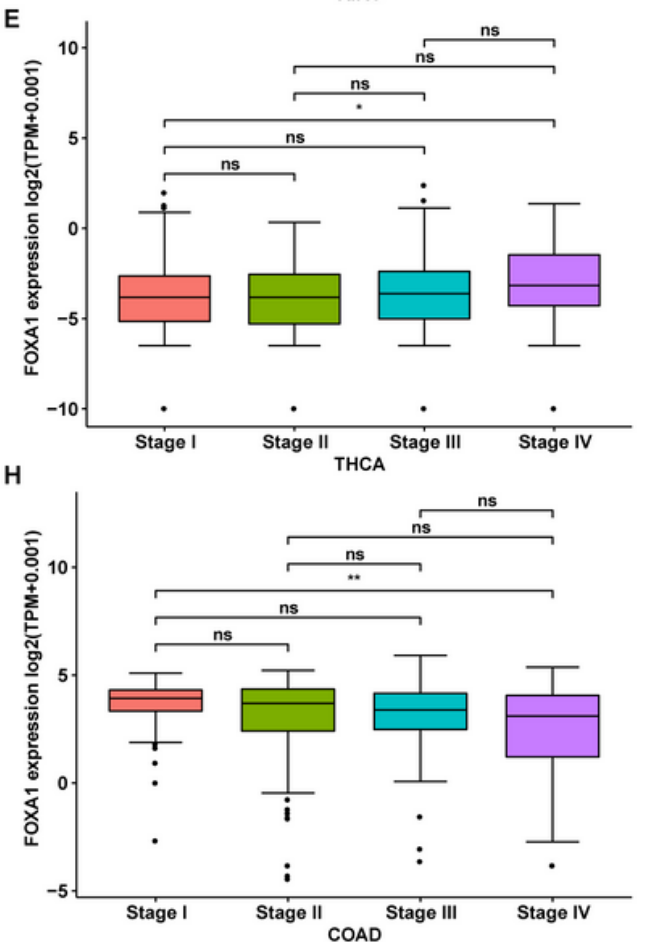

C
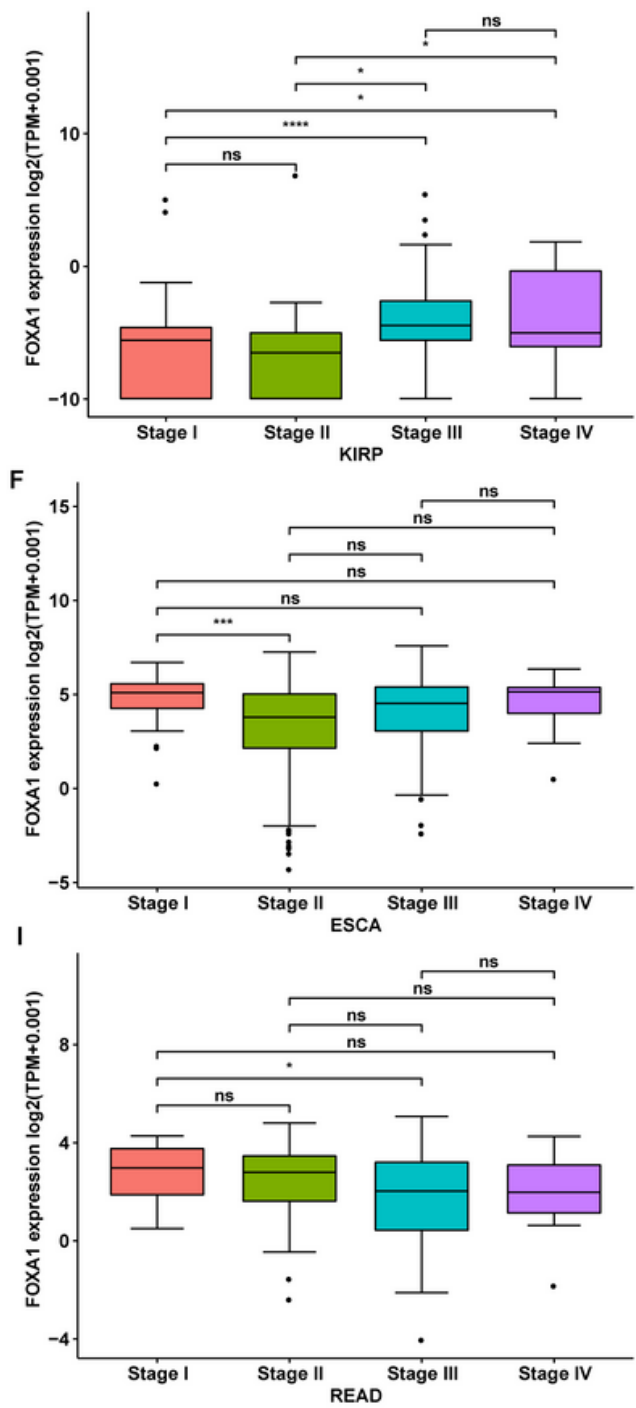

Figure 2

FOXA1 expression in different tumor stages. (A-I) The FOXA1 expression in different tumor stages in indicated tumor stages from TCGA cohort. 
A
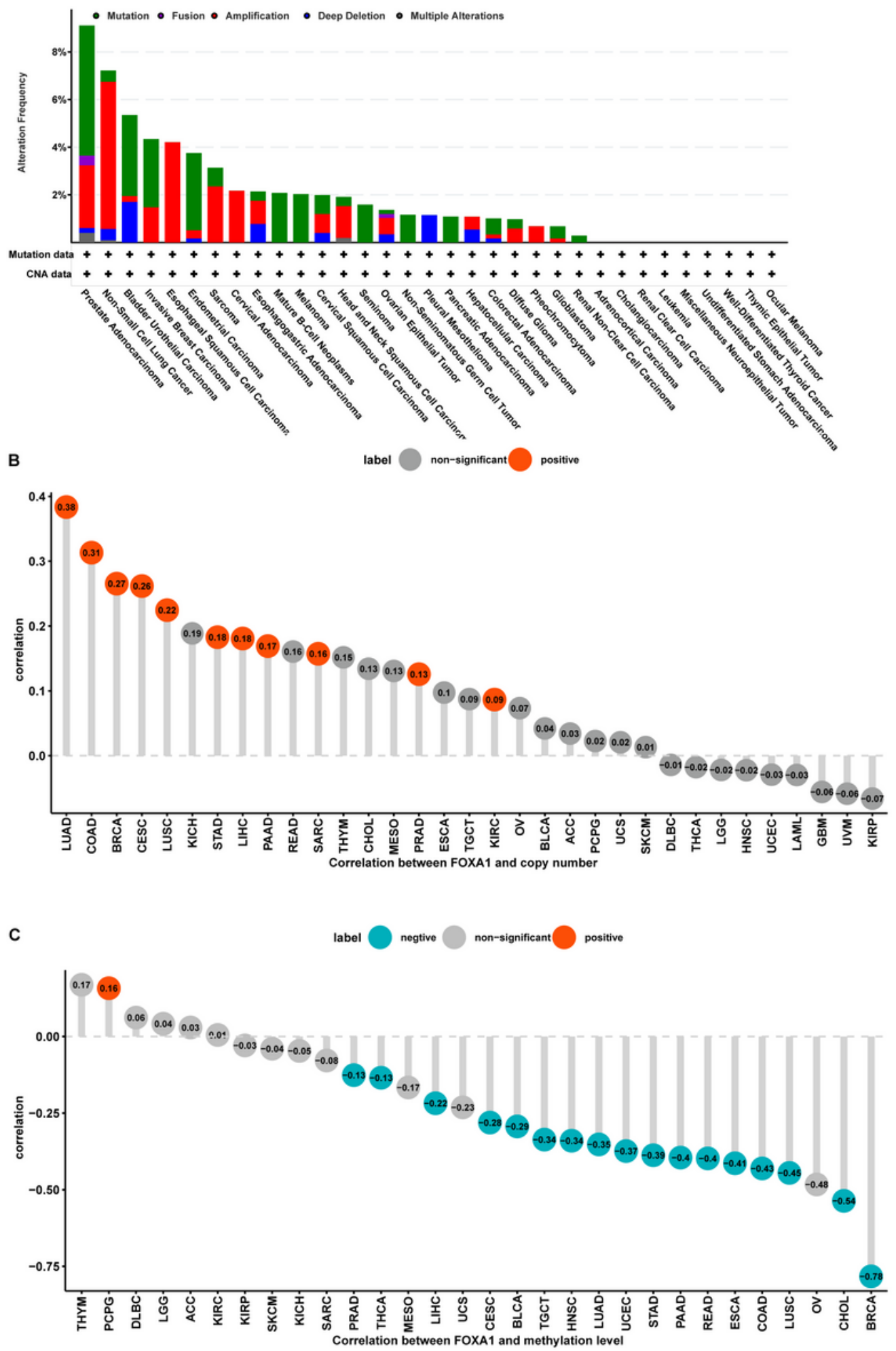

Figure 3

Gene alteration of FOXA1. (A) The genetic alteration of FOXA1 in TCGA pan-cancer. (B) The correlation between FOXA1 expression and copy number in TCGA pan-cancer. (C) The correlation between FOXA1 expression and methylation level in TCGA pan-cancer. 
A
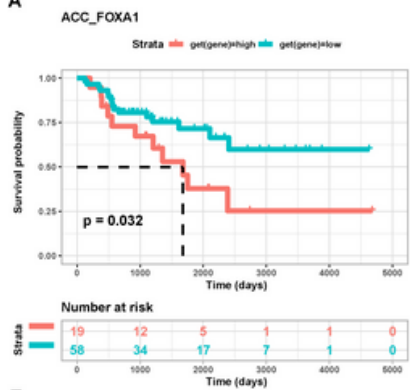

E LGG_FOXA1
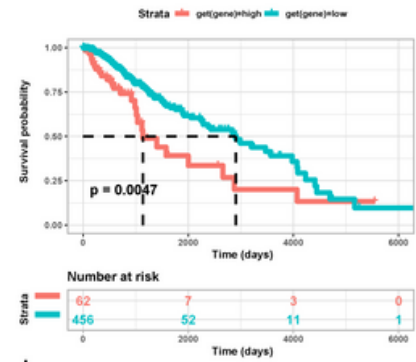

I THCA_FOXA1

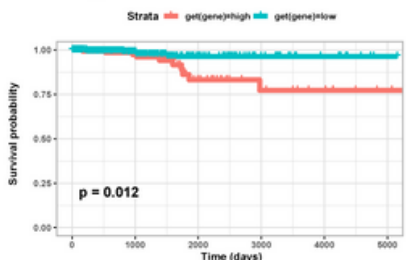

$\mathbf{y}=\begin{aligned} & \text { Time (days) } \\ & \text { Number at risk }\end{aligned}$
B
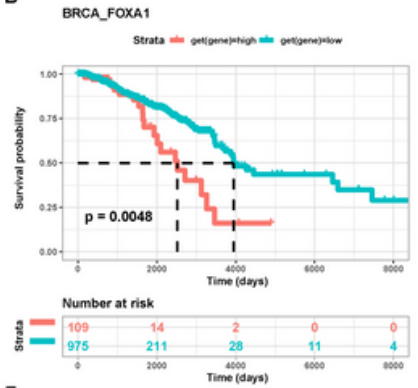

F MESO_FOXA1
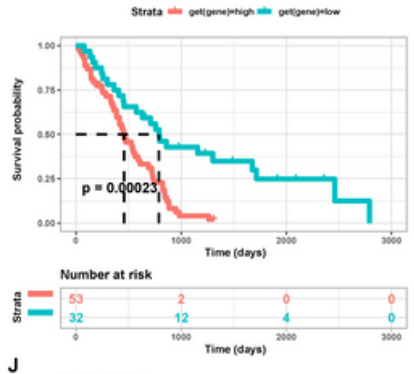

J BLCA_FOXA1

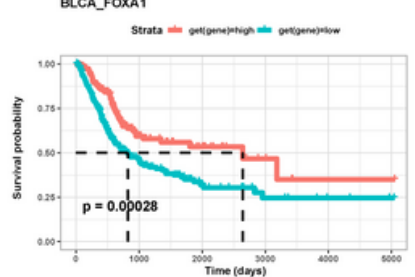

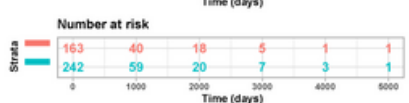

c
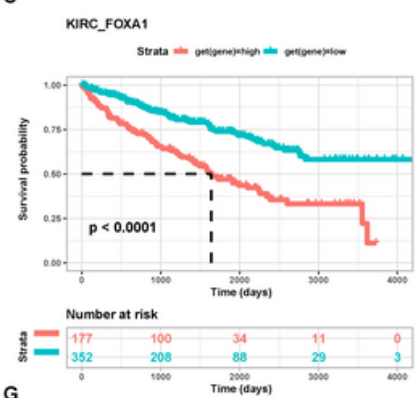

G SARC_FOXA1
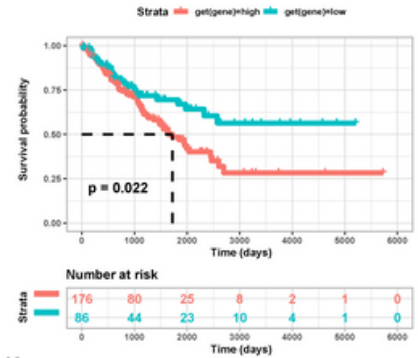

K COAD_FOXA1

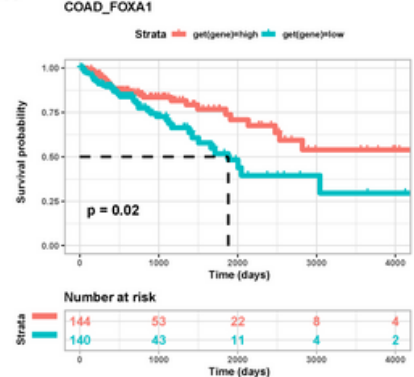

D KIRP FOXA1
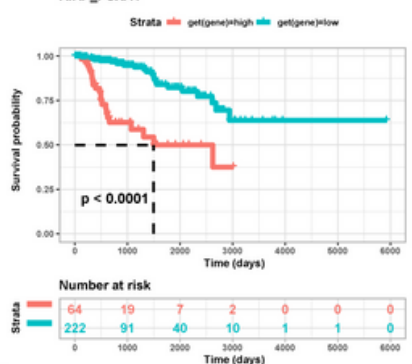

H SKCM_FoxA
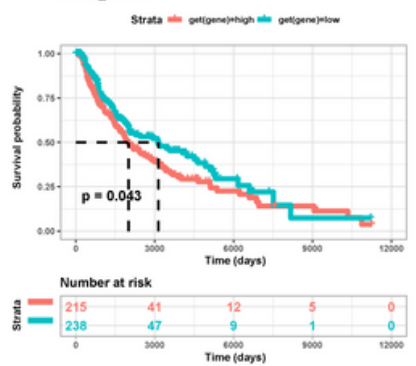

L PAAD_FOXA1

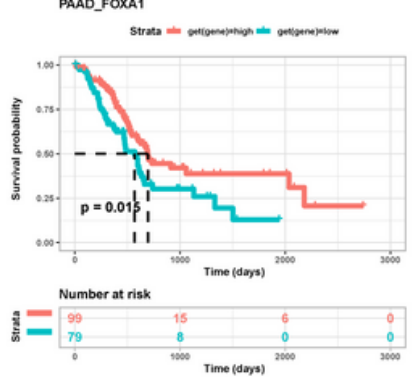

Figure 4

Prognostic value of FOXA1. (A-L) The Kaplan-Meier analyses of FOXA1 in indicated tumor types. The optimum cutoff value of FOXA1 in each tumor type was set. 

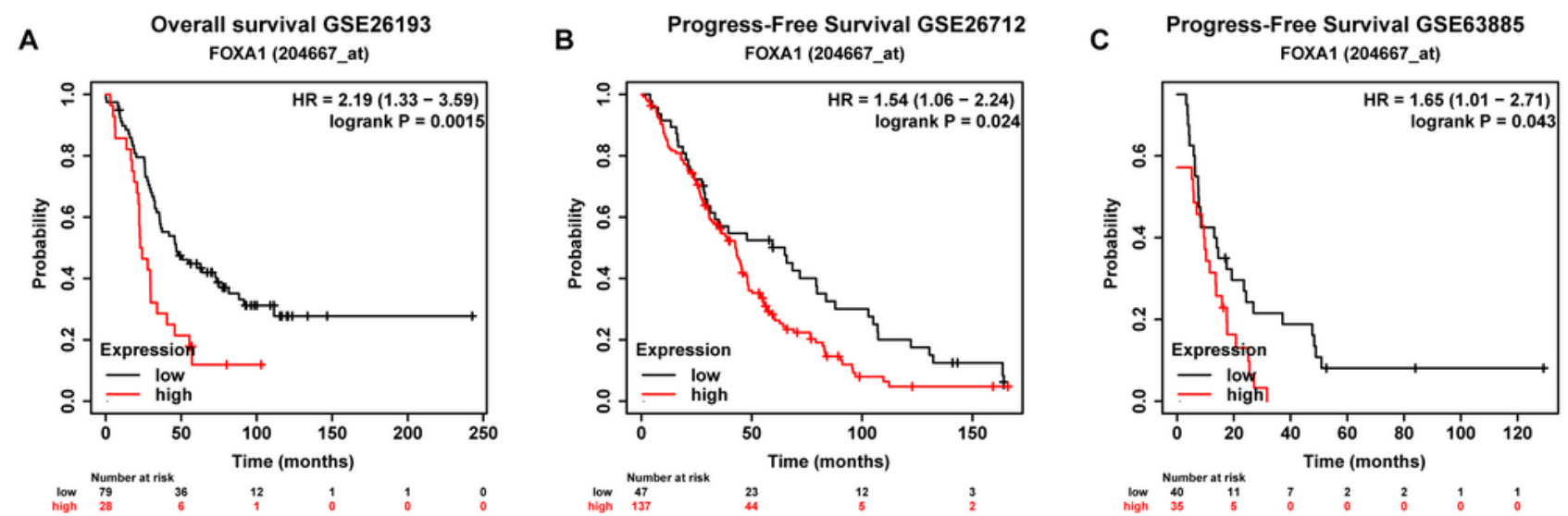

D

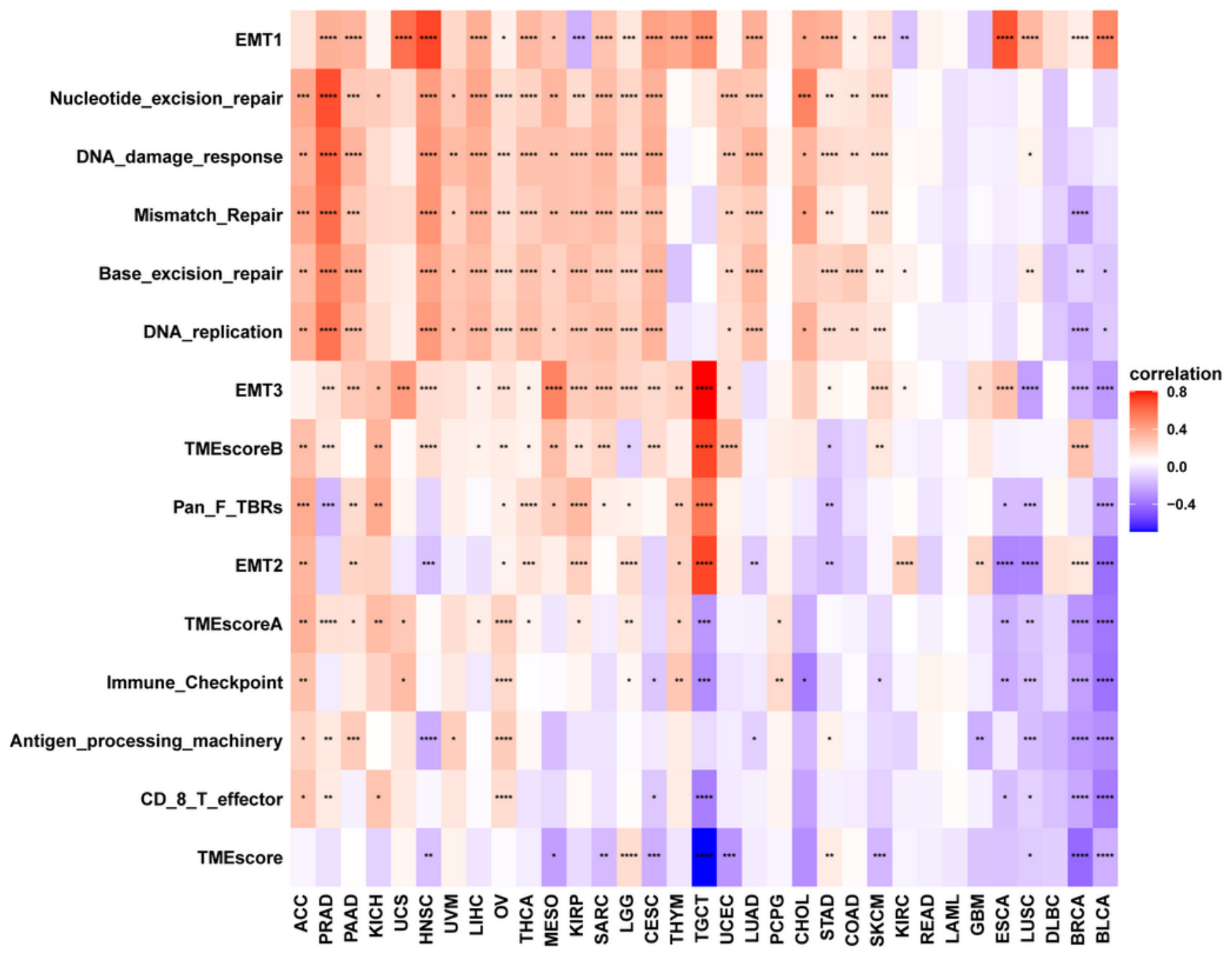

Figure 5

TME analysis. (A-C) The Kaplan-Meier analyses of FOXA1 in EOC datasets, including GSE26193 (A), GSE26712 (B), and GSE63885 (C). The optimum cutoff value of FOXA1 in each dataset was set. (D) The correlation between TME-related signature scores and FOXA1 expression. 

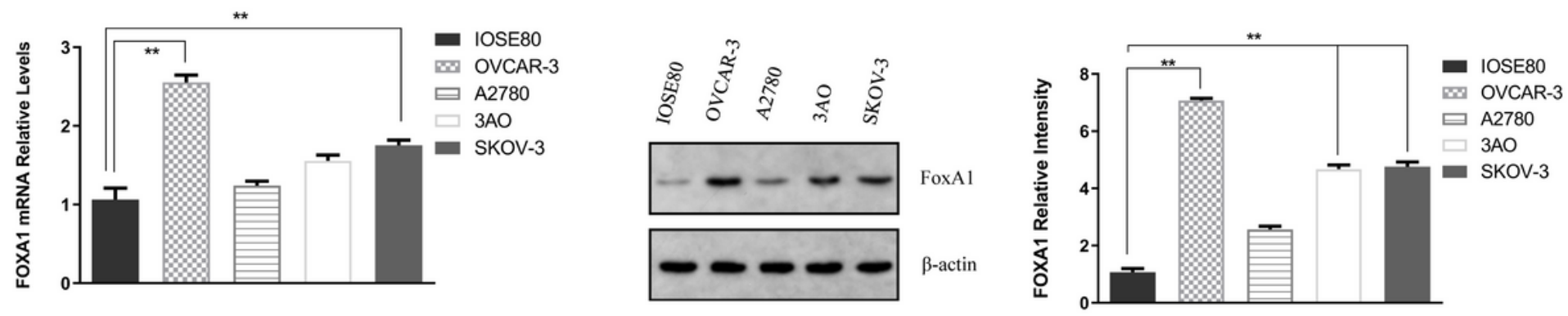

D

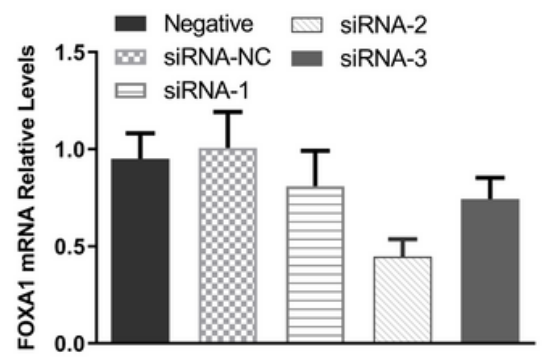

E

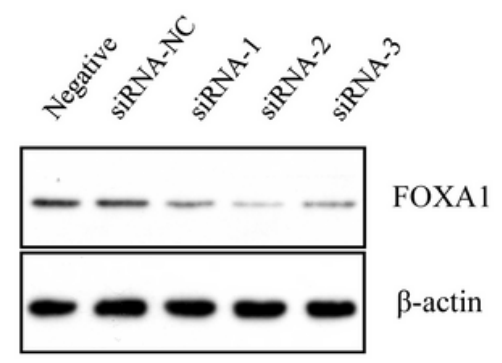

$\mathbf{F}$

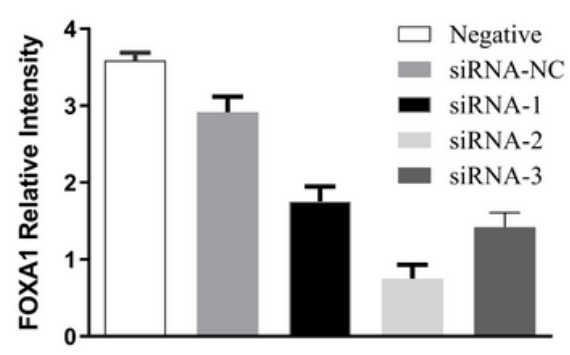

Figure 6

FOXA1 expression was upregulated in ovarian cancer cells. (A-C) FOXA1 expression was assessed by qRT-PCR (A) and western blot (B) in IOSE80 (human ovarian epithelial cell line from normal tissues) and OVCAR-3/A2780/3AO/SKOV-3 cells (human ovarian cancer cell lines). Relative mRNA and protein levels of FOXA1 were quantified in (A) and (C); $N=3$, two-way ANOVA. (D-E) FOXA1 knockdown via transient transfection with siRNA-2 in OVCAR-3 cell was confirmed by qRT-PCR (D) and western blot (E). siRNA-NC transfected cells were set as the control. The experiments were repeated independently for 3 times. 
A

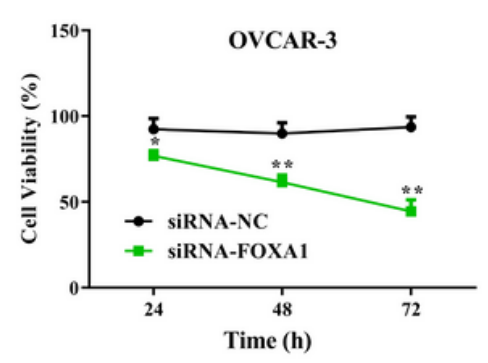

B

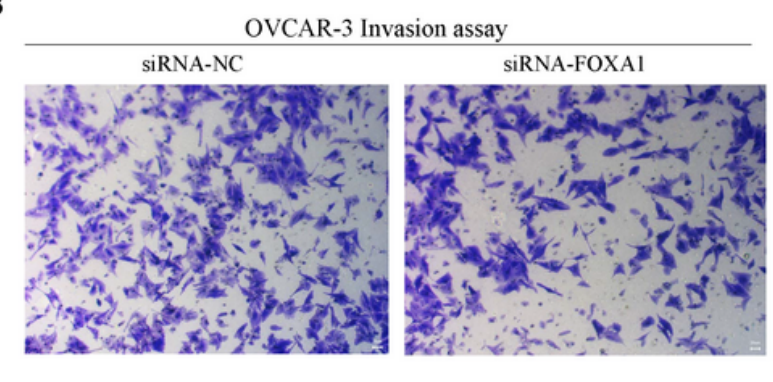

C

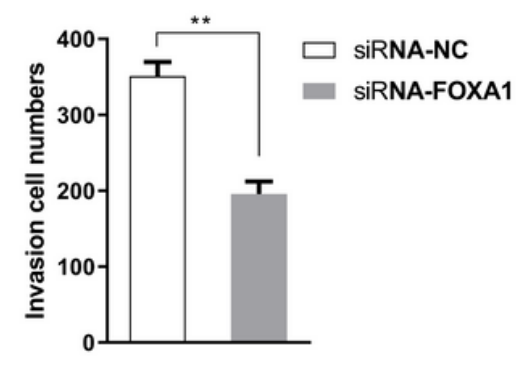

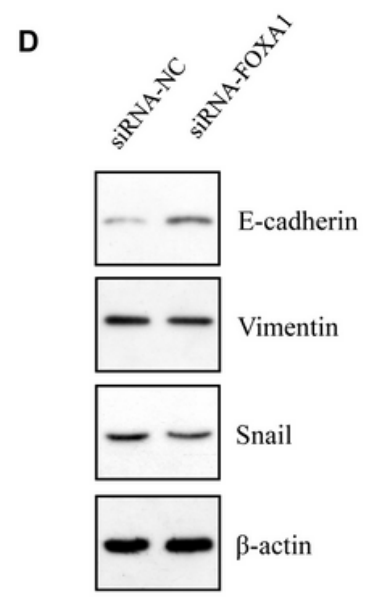

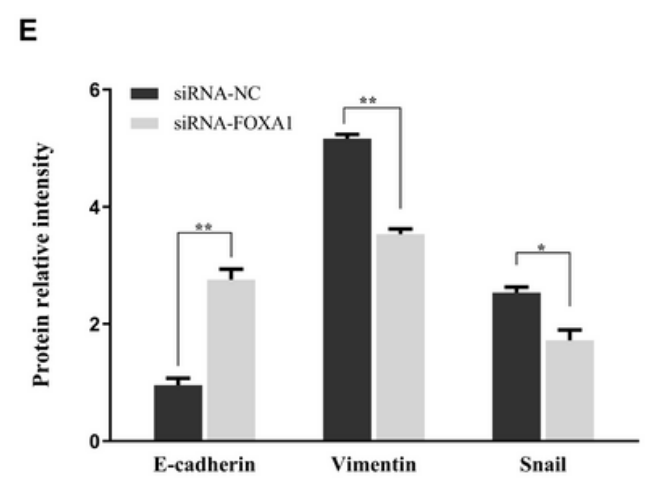

F

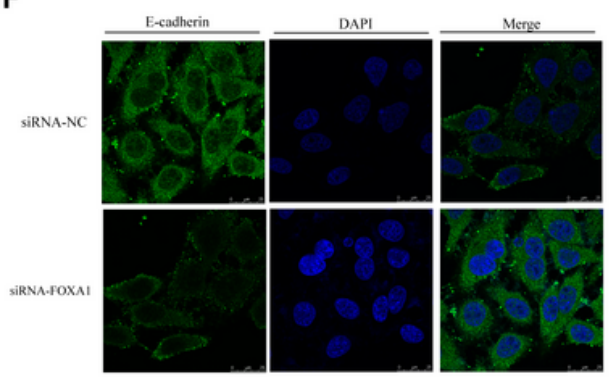

G

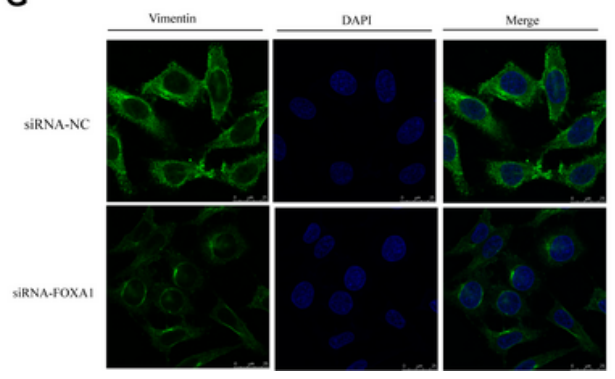

\section{Figure 7}

Effects of FOXA1 silencing on OVCAR-3 cell proliferation and invasion. (A) The proliferation was measured by CCK8 assay at 24, 48 and $72 \mathrm{~h}$ respectively. The results were obtained from the 6 replicates in each group and presented as average value \pm SD. (B) The invasiveness of OVCAR-3 cells was detected by the transwell migration assay. Representative images were selected (magnification $\times 100)$. (C) The number of invaded cells were quantified and obtained from three independent experiments. (D) Protein levels of E-cadherin, vimentin and snail were measured by Western blot after $48 \mathrm{~h}$ following siRNA transfection. (E) Quantifications of representative blots were demonstrated in bar graphs. Protein levels of E-cadherin, vimentin and snail in the FOXA1-silenced group (siRNA-FOXA1) were compared to those in the control group (siRNA-NC) and presented in the form of average \pm SD. (E-F) Representative immunofluorescence staining for E-cadherin $(E)$ and vimentin $(F)$ in the siRNA-NC- or siRNA-FOXA1transfected OVCAR3 cells. (Original magnification $\times 630$ ). 
A

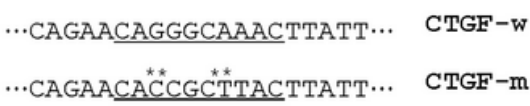

B

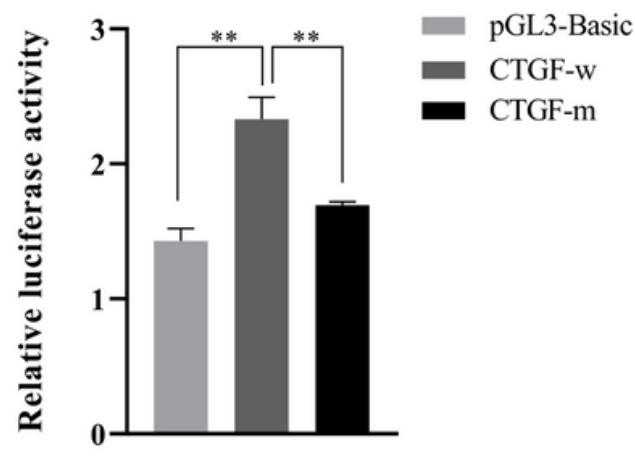

C
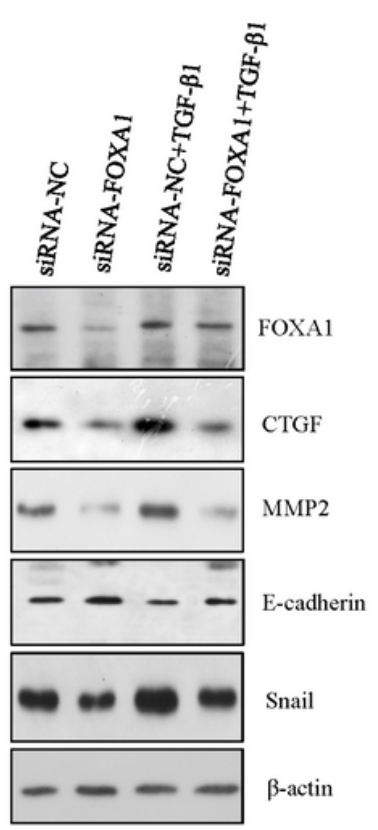

D
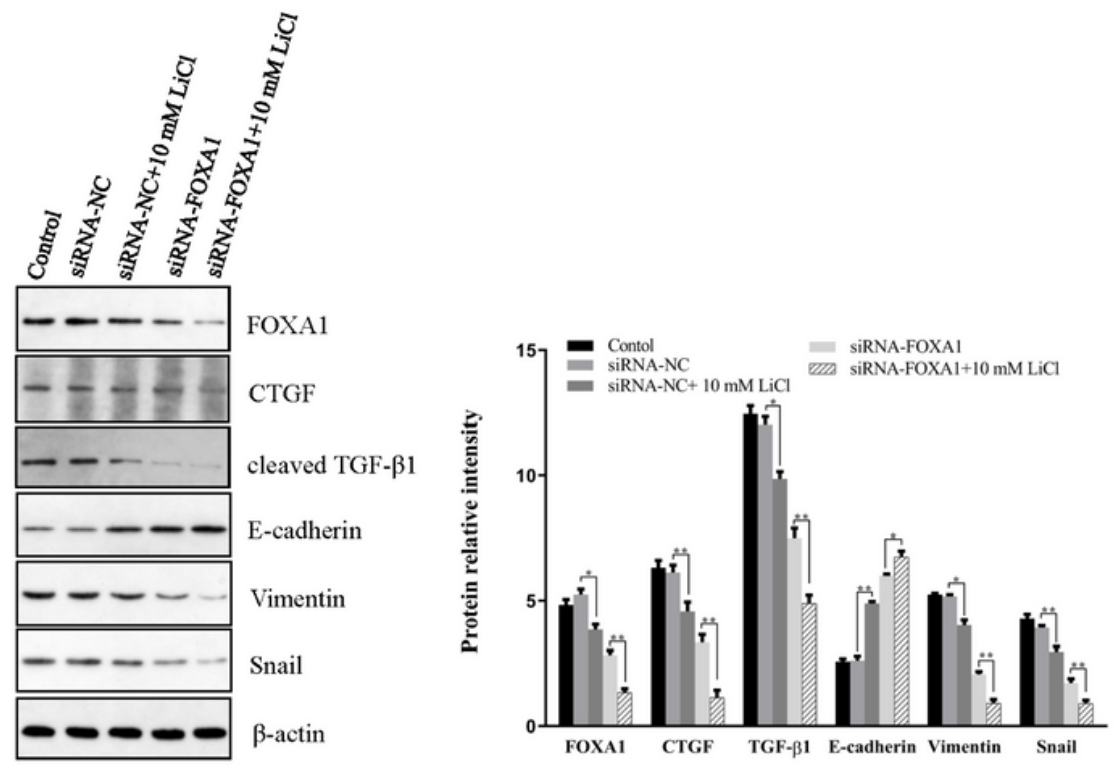

Figure 8

The suppressive effects of FOXA1-silencing on EMT through TGF-beta 1 signaling pathway. (A) Predicted binding site of FOXA1 at the wild-type CTGF promoter region and the mutant CTGF promoter (-399 to -390) were present; (B) Relative luciferase activity was presented as per the ratio of the intensity of firefly luciferase to that of renilla. (C) Western blot analysis on protein expression levels of FOXA1, CTGF, MMP2, E-cadherin, and Snail in the siRNA-NC-, or the siRNA-FOXA1-transfected cells treated with or without TGF- $\beta 1$. Quantifications of protein expressions represent the mean \pm standard deviation (SD) of the results from six independent replicates in each group. (D) Expressions of FOXA1, CTGF, cleaved TGF-beta 1 and EMT-associated markers including E-cadherin, vimentin and snail were analyzed by western blot after $48 \mathrm{~h}$ after the transfected cells treated with or without lithium chloride. Quantification of the 
representative blots was represented as the mean \pm standard deviation (SD) of the results from three independent experiment in bar graphs.

A

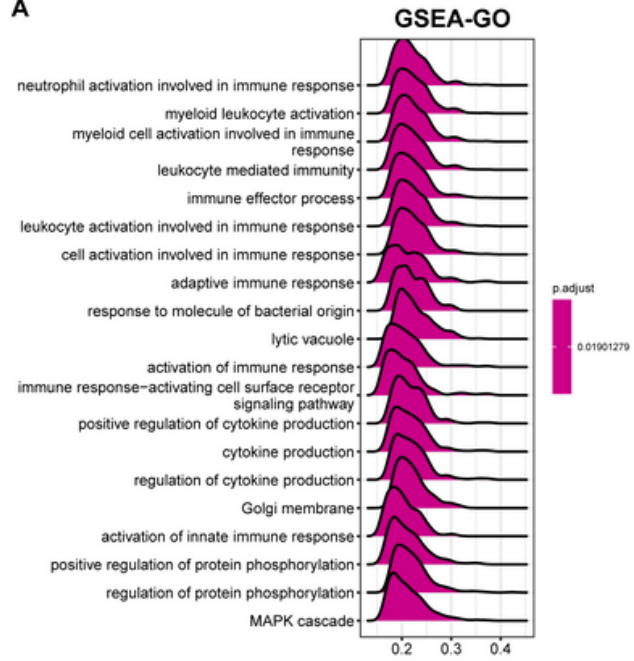

B

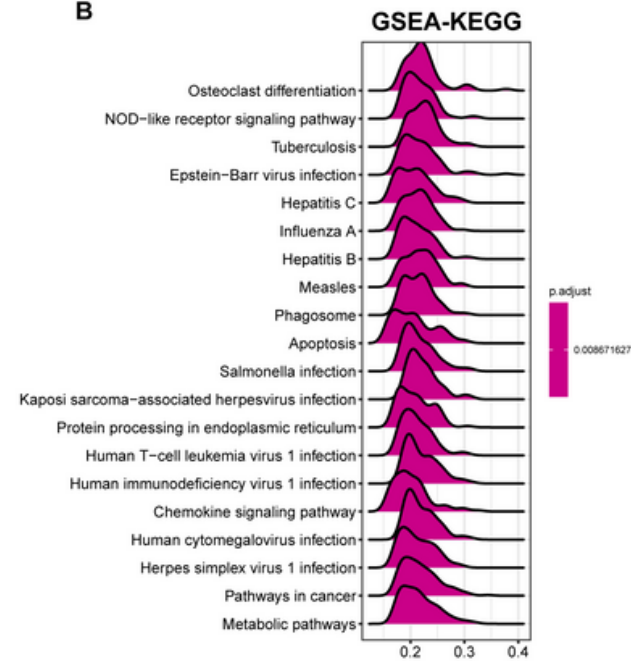

C

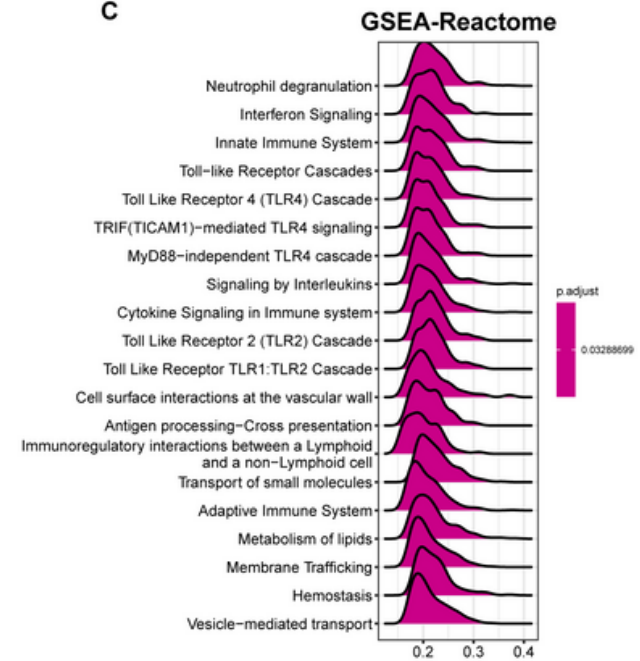

\section{Figure 9}

GSEA od FOXA1. (A-C) The GSEA results of FOXA1 in TCGA-OV cohort, including GSEA-GO. (A), GSEAKEGG (B), and GSEA-Reactome (C). 

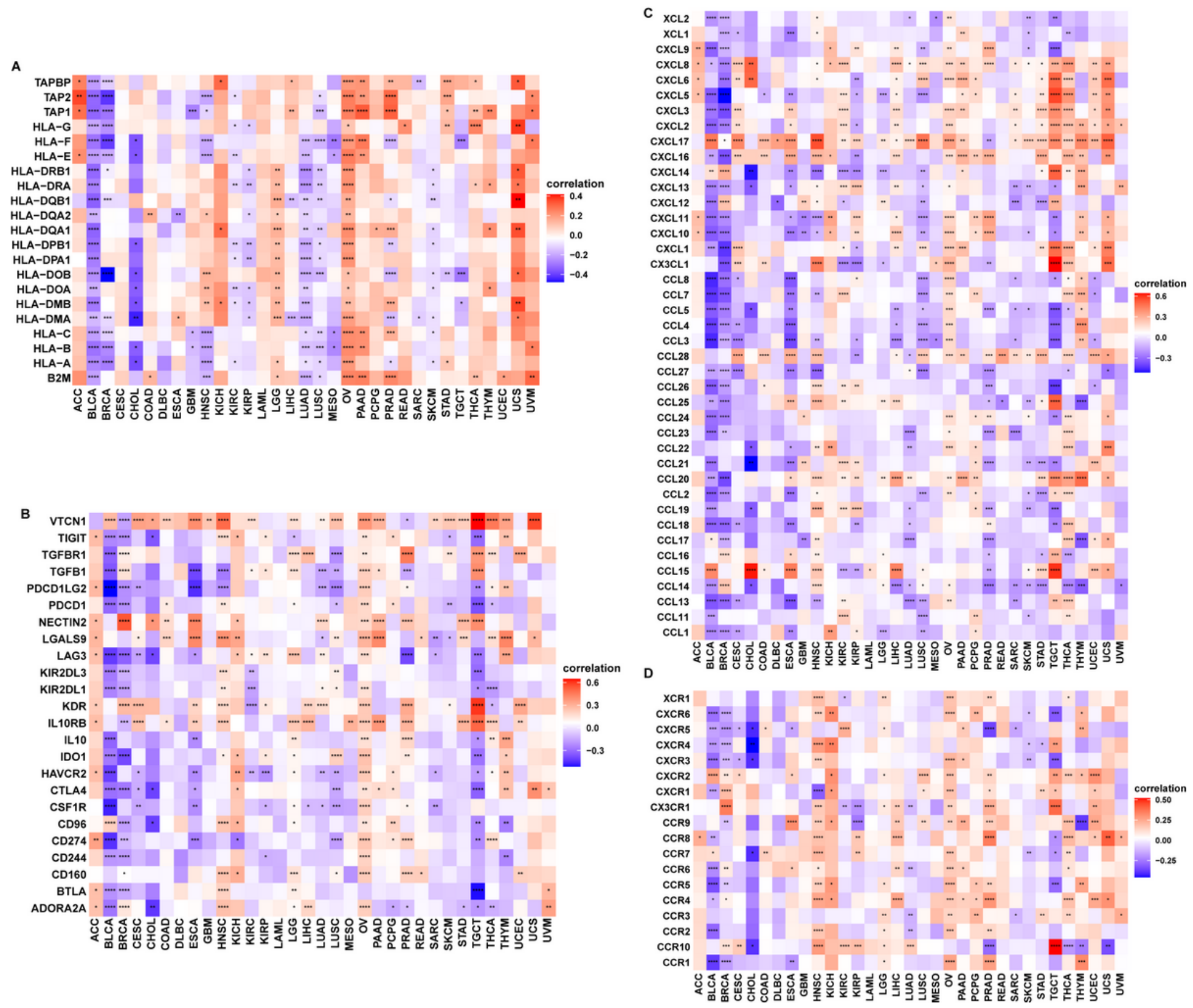

Figure 10

The correlation analysis. (A) The correlation between MHC genes and FOXA1. (B) The correlation between immunosuppressive genes and FOXA1. (C) The correlation between immune activating genes and FOXA1. (D) The correlation between chemokine receptors and FOXA1. 


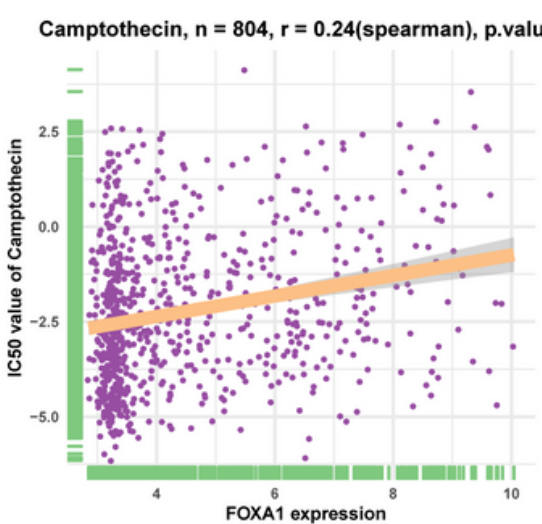

Cytarabine, $n=749, r=0.26$ (spearman), p.value $=0$

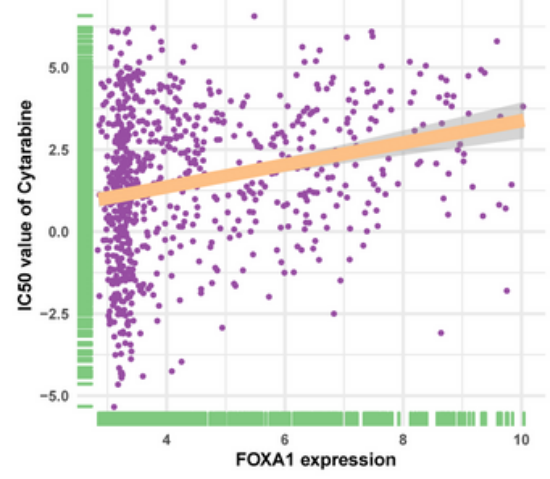

Vorinostat, $n=755, r=0.21$ (spearman), p.value $=0$

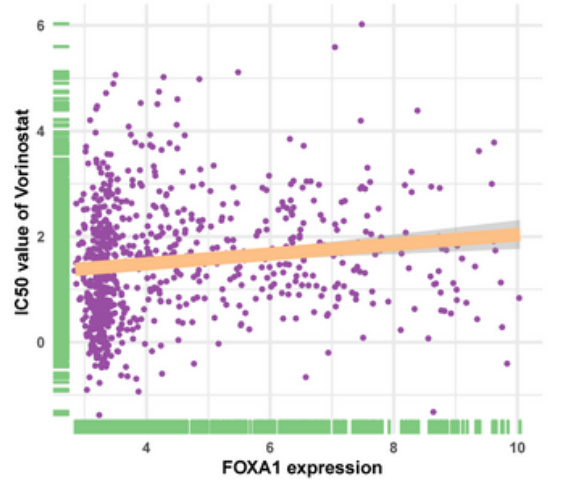

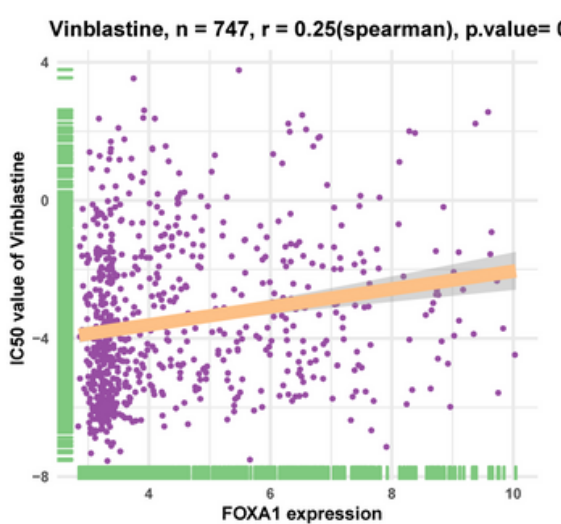

Docetaxel, $n=1430, r=0.15($ spearman $)$, p.value $=0$

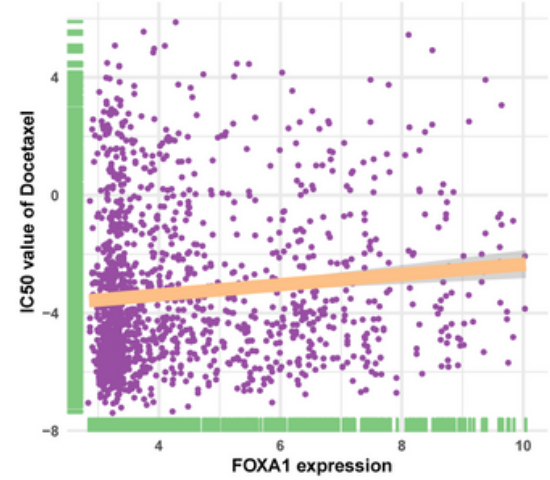

Nilotinib, $n=754, r=0.21$ (spearman), p.value $=0$

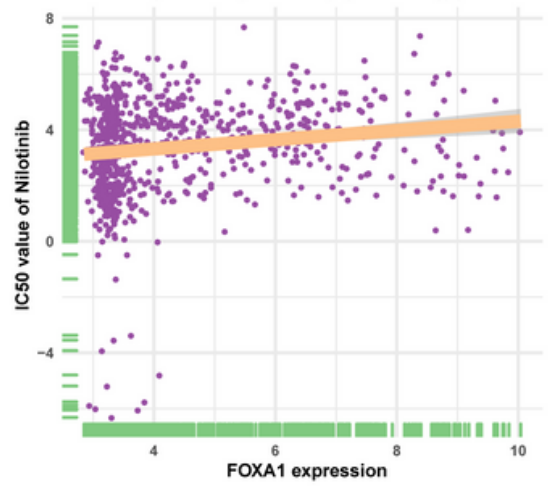

Cisplatin, $n=765, r=0.25$ (spearman), p.value $=0$

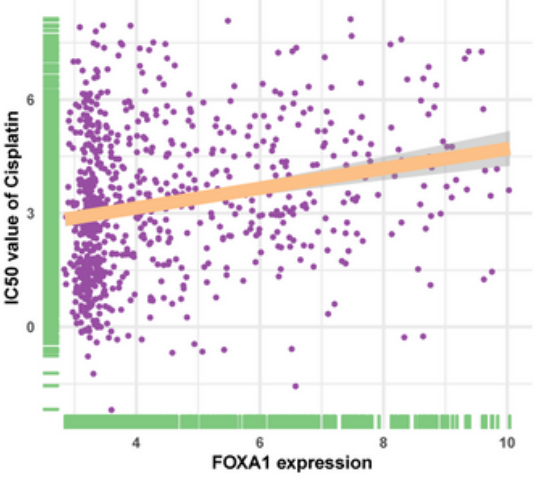

Navitoclax, $n=747, r=0.08$ (spearman), p.value $=0.029$;

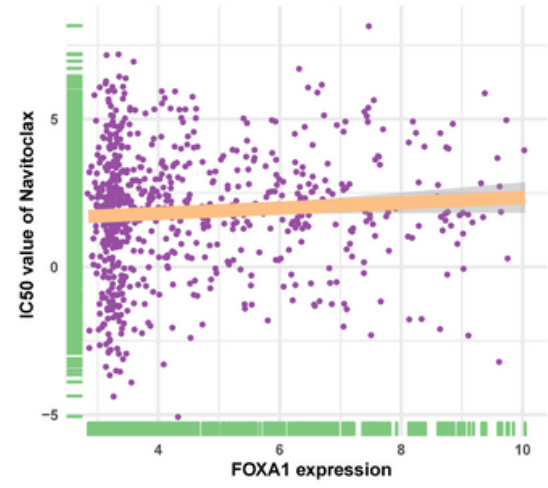

Olaparib, $\mathrm{n}=758, \mathrm{r}=0.21$ (spearman), p.value $=0$

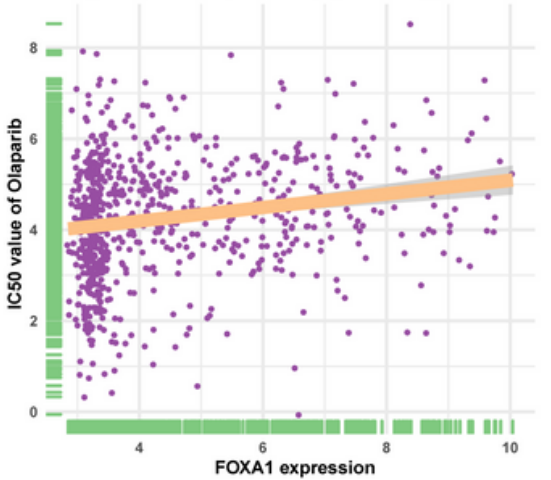

Figure 11

The correlation analysis between FOXA1 and IC50 values of indicated anti-cancer drugs. 